\title{
Segregation, recirculation and deposition of coarse particles near two-dimensional avalanche fronts
}

\author{
J. M. N. T. GRA $\mathrm{Y}^{1} \dagger$ AND C. ANCEY ${ }^{2}$ \\ ${ }^{1}$ School of Mathematics and Manchester Centre for Nonlinear Dynamics, University of Manchester, \\ Oxford Road, Manchester M13 9PL, UK \\ ${ }^{2}$ École Polytechnique Fédérale de Lausanne, Ecublens, 1015 Lausanne, Switzerland
}

(Received 14 May 2008 and in revised form 5 February 2009)

Stratification patterns are formed when a bidisperse mixture of large rough grains and smaller more mobile particles is poured between parallel plates to form a heap. At low flow rates discrete avalanches flow down the free surface and are brought to rest by the propagation of shock waves. Experiments performed in this paper show that the larger particles are segregated to the top of the avalanche, where the velocity is greatest, and are transported to the flow front. Here the particles are overrun but may rise to the free surface again by size segregation to create a recirculating coarse-grained front. Once the front is established composite images show that there is a steady regime in which any additional large grains that reach the front are deposited. This flow is therefore analogous to finger formation in geophysical mass flows, where the larger less mobile particles are shouldered aside to spontaneously form static lateral levees rather than being removed by basal deposition in two dimensions. At the heart of all these phenomena is a dynamic feedback between the bulk flow and the evolving particle-size distribution within the avalanche. A fully coupled theory for such segregation-mobility feedback effects is beyond the scope of this paper. However, it is shown how to derive a simplified uncoupled travellingwave solution for the avalanche motion and reconstruct the bulk two-dimensional flow field using assumed velocity profiles through the avalanche depth. This allows a simple hyperbolic segregation theory to be used to construct exact solutions for the particle concentration and for the recirculation within the bulk flow. Depending on the material composition and the strength of the segregation and deposition, there are three types of solution. The coarse-particle front grows in length if more large particles arrive than can be deposited. If there are fewer large grains and if the segregation is strong enough, a breaking size-segregation wave forms at a unique position behind the front. It consists of two expansion fans, two shocks and a central 'eye' of constant concentration that are arranged in a 'lens-like' structure. Coarse grains just behind the front are recirculated, while those reaching the head are overrun and deposited. Upstream of the wave, the size distribution resembles a small-particle 'sandwich' with a raft of rapidly flowing large particles on top and a coarse deposited layer at the bottom, consistent with the experimental observations made here. If the segregation is weak, the central eye degenerates, and all the large particles are deposited without recirculation.

$\dagger$ Email address for correspondence: nico.gray@manchester.ac.uk 


\section{Introduction}

Bouldery flow fronts develop in many different kinds of hazardous geophysical mass movements, including debris flows (Costa \& Williams 1984; Pierson 1986; Iverson 2003, 2005), lahars (Vallance 2000), wet snow avalanches (Jomelli \& Bertran 2001), rock slides (Cruden \& Hungr 1986; Bertran 2003) and pyroclastic flows (Calder, Sparks \& Gardeweg 2000; Iverson \& Vallance 2001). In the high-solids-volumefraction regions of these flows, the large particles commonly segregate towards the surface, where the velocity is greatest, and are transported to the margins where they accumulate. Once the large grains reach the front they may either be overrun by the flow front and rise quickly to the surface again by particle-size segregation, to create a recirculating path (Pierson 1986; Pouliquen, Delour \& Savage 1997), or, if the particles are too large to be overrun, simply be pushed en masse in front of the flow (Pouliquen \& Vallance 1999). In debris flows the mobility is conferred by high basal pore pressures that reduce the frictional resistance to motion (Iverson 1997). Since the pore pressure is dissipated much more rapidly amidst the coarse clasts than in the finer-grained material, the bouldery margins experience much greater frictional resistance to motion than the flow interior. This can lead to the spontaneous development of surge waves on steep slopes. On shallower slopes, where the large particles are able to come to rest, the more resistive bouldery material is shouldered to the sides to form stationary lateral levees that channelize the finer-grained interior and enhance the overall run-out distance (Iverson \& Vallance 2001; Iverson 2003, Iverson 2005).

Bouldery lateral levees are a ubiquitous feature of mass flows and granular avalanches in general. Pouliquen et al. (1997), Pouliquen \& Vallance (1999), Félix \& Thomas (2004), Aranson, Malloggi \& Clement (2006) and Goujon, DallozDubrujeaud \& Thomas (2007) have generated a fingering instability in dry granular flows in the laboratory by pouring a mixture of large irregular and smaller rounded particles down a rough inclined plane. As the large particles collect at a flow front, it rapidly deforms and breaks into a series of fingers, with the larger grains at the head being shouldered aside into slower moving/static lateral levees, in an analogous manner to debris flows. In all of these flows the evolving particle-size distribution provides feedback to the bulk motion, with the larger less mobile grains experiencing greater resistance to motion than the smaller ones. In this paper this is termed a segregation-mobility feedback. One way of developing mathematical models that take account of mobility differences between the particles is to couple a depth-averaged avalanche model (e.g. Grigorian, Eglit \& Iakimov 1967; Savage \& Hutter 1989; Iverson 1997; Gray, Wieland \& Hutter 1999; Pouliquen 1999a,b; Wieland, Gray \& Hutter 1999; Denlinger \& Iverson 2001; Iverson \& Denlinger 2001; Gray, Tai \& Noelle 2003; Bartelt, Buser \& Platzer 2007; Cui, Gray \& Johannesson 2007; Gray \& Cui 2007) with a model for size segregation (e.g. Savage \& Lun 1988; Dolgunin \& Ukolov 1995; Gray \& Thornton 2005; Gray \& Chugunov 2006; Thornton, Gray \& Hogg 2006). The feedback between the two can be achieved by $(a)$ using a composition-dependent basal friction law (e.g. Pouliquen \& Vallance 1999), (b) modifying the assumed velocity profile through the avalanche depth (e.g. Phillips et al. 2006; Linares-Guerrero, Goujon \& Zenit 2007; Rognon et al. 2007) or (c) making the deposition dependent on the evolving particle-size distribution. A fully coupled theory is beyond the scope of this paper, but, motivated by observations from two-dimensional stratification experiments (Williams 1968; Makse et al. 1997; Gray \& Hutter 1997, 1998; Baxter et al. 1998; Herrmann 1998), the key steps that are necessary to reconstruct the bulk flow field $\boldsymbol{u}$ from the depth-averaged velocity 
$\overline{\boldsymbol{u}}$ are illustrated, and exact solutions for the particle-size distribution in the resulting flow are derived.

\subsection{Stratification-pattern experiments}

Stratification patterns form when a mixture of larger rougher grains and smaller smoother grains are poured at low flow rates into a narrow gap between two parallel plates to build up a heap (Williams 1968; Gray \& Hutter 1997, 1998; Makse et al. 1997; Baxter et al. 1998; Herrmann 1998). The pattern consists of alternating bands, or stripes, of large and small particles that are laid down by avalanches that flow down the free surface of the heap. If, instead, the larger particles are smoother than the fine ones, the flow changes completely, and the large particles 'segregate' into the triangular region of the pile that is the farthest from the source (Herrmann 1998). This provides strong evidence that the segregation-mobility feedback effect controls both the dynamics of the avalanche and the nature of the deposition. The flow rate is also important (Gray \& Hutter 1997; Baxter et al. 1998; Gray \& Tai 1998). This is made particularly clear in thin rotating drum experiments (Gray \& Hutter 1997), where at slow rotation rates Catherine wheel patterns form, in the intermittent avalanching regime. At higher rotation rates, in the continuous rolling regime, a radial segregation pattern develops, except near $50 \%$ fill, where petals form through longer time-scale wave interactions (Hill, Gioia \& Amaravadi 2004; Zuriguel et al. 2006).

A thin hopper, originally made by Gray \& Hutter (1997, 1998), has been used to take a closer look at the dynamics of the flow, using a fast digital camera. A photograph of the hopper is shown in Gray \& Hutter's (1997) figure 3. It consists of two perspex plates $(70 \mathrm{~cm}$ high $\times 37 \mathrm{~cm}$ wide) that are separated by aluminium spacers along the sidewalls to produce a gap of $3 \mathrm{~mm}$. The base is made of perspex, and there is an orifice at the centre that allows the hopper to be emptied. The experiment is continuously fed from a cylindrical hopper, which is balanced on top of the apparatus near one of the aluminium sidewalls. For the experiments presented here, the hopper is filled with a 50:50 mix by volume of large white sugar crystals of size 500-600 $\mu \mathrm{m}$ and small dark iron spheres of size $210-420 \mu \mathrm{m}$. A narrow stream of grains was then allowed to fall down between the parallel plates to build up a pile of grains. After a short while a flow develops, in which (i) the incoming grains gather at the top of the heap and intermittently collapse (ii) sending an avalanche down the surface of the pile, (iii) until the front hits the base plate or the sidewall, and (iv) a normal shock wave, or granular bore (e.g. Gray \& Hutter 1997; Gray \& Tai 1998; Gray et al. 2003), propagates up through the avalanche (v) bringing all the grains to rest. The processes (i)-(v) are then repeated, except that the old free surface becomes the new basal surface for the next avalanche to flow down.

The digital camera was used to acquire close-up images of the flow as the stratification pattern developed. The camera was placed approximately midway between the source and the downstream wall and about $15 \mathrm{~cm}$ above the base. Figure 1 shows a sequence of images taken as a single avalanche flows down the free surface of the pile. The initial static deposit is shown in figure 1( $a$ ), and the free surface is located at the top of the uppermost layer of large white particles and lies at an angle of approximately $36^{\circ}$. In figure $1(c-g)$ a coarse-particle-rich flow front propagates down the free surface, at approximately constant speed, and behind it a steady uniform-thickness flow rapidly establishes itself. Within the interior of the avalanche the particles are strongly inversely graded, with the large particles on top of the fine ones, but just behind the flow front there is a mixed region in which segregation takes place. In figure $1(p-s)$ a diffuse normal shock propagates up through 


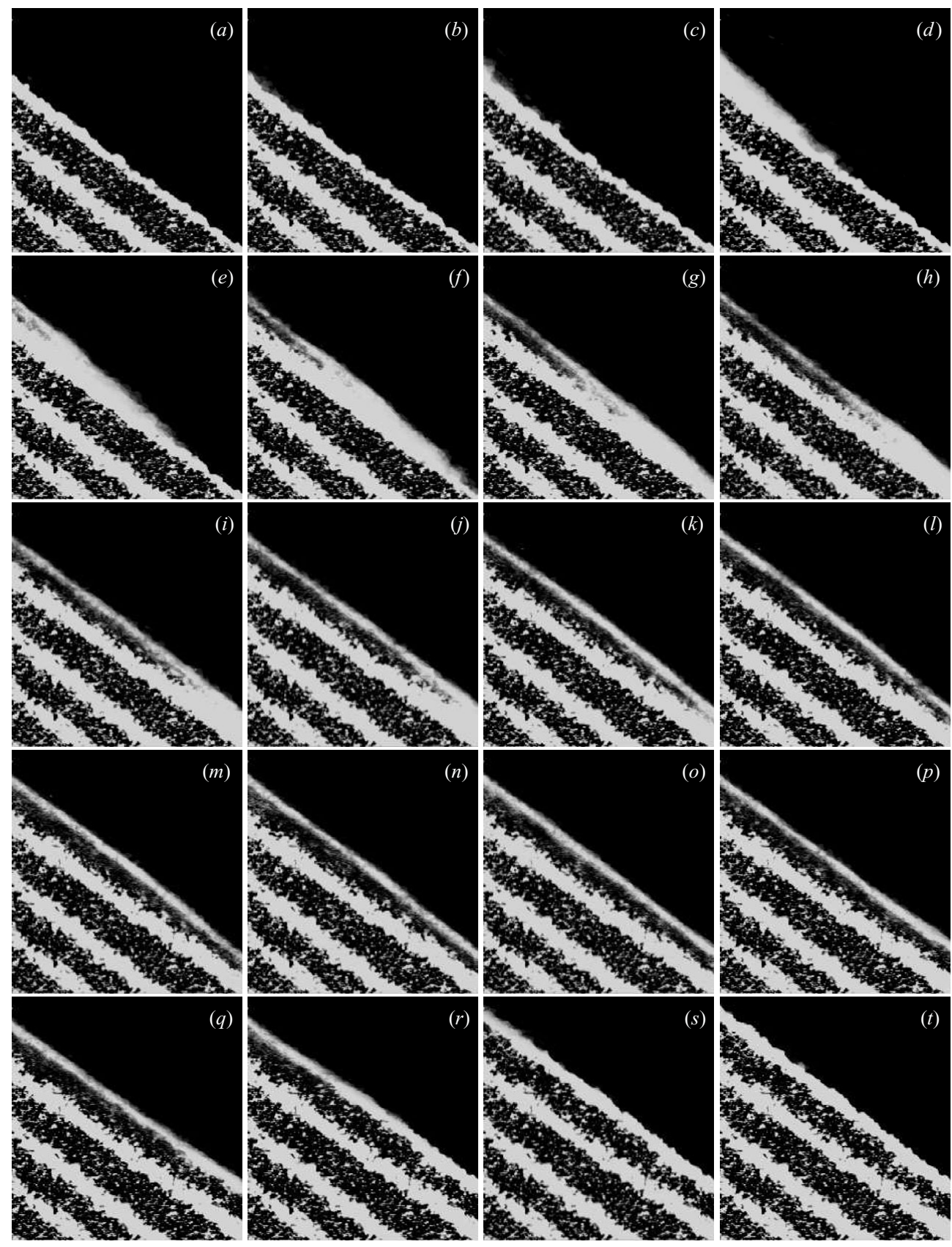

FIGURE 1. A sequence of images $(a)-(t)$ showing how a stratification pattern is built up in a deposited heap by the passage of a single avalanche. The flow occurs in the narrow gap between two parallel plates with a spacing of $3 \mathrm{~mm}$; each image is $36.1 \mathrm{~mm}$ wide and $38.2 \mathrm{~mm}$ high. The mixture is composed of large rough sugar crystals (white) and smaller more mobile spherical iron particles, which appear generally darker but have some reflections that give them a grainy texture. Subsequent avalanches build up further striped layers; an animation showing the flow and deposition of two avalanches is available with the online version of the paper. 


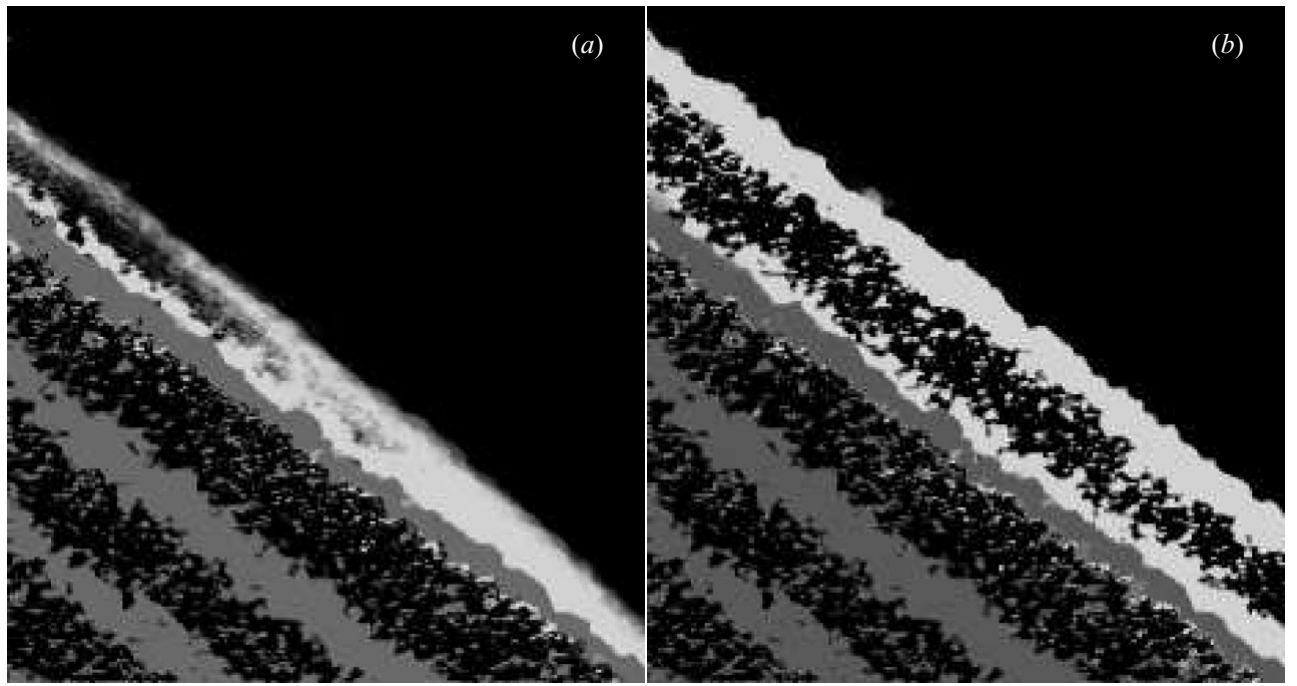

FIGURE 2. Composite images of the stratification pattern emphasizing the grains close to the free surface that form the current avalanche. They are derived by darkening the stationary deposit shown in figure $1(a)$ and overlaying it on figures $1(g)$ and $1(t)$, respectively. $(a)$ The structure of the coarse-particle-rich flow front and the inversely graded flow behind; $(b)$ The final emplacement once the shock wave has brought the grains to rest.

the avalanche, bringing the grains to rest and freezing the inversely graded layers into the deposit to create a static stripe of large and small particles. A longer animation sequence showing the passage of two avalanches is available with the online version of the paper.

A new feature of these flows, which has not been previously reported and is not immediately apparent is the existence of a thin layer of large particles that are deposited at the base of the avalanche as it propagates downslope. The basal deposition is made visible by darkening the image of the initial stationary deposit shown in figure $1(a)$ and overlaying it on later images of the avalanche. Figure 2(a) shows the structure of the flow front, with the coarse particles forming part of the current flow highlighted in white. The head of the flow is entirely composed of large particles, but further upstream the combination of flow and deposition creates a particle-size distribution that resembles a small-particle 'sandwich', with a raft of rapidly flowing large particles on top and a coarse carpet of static grains at the bottom. Figure 2(b) shows the final static deposit after the normal shock wave has brought the grains to rest. The thickness of the deposited carpet is unaffected by the passage of the shock, but the upper layer of large grains thickens significantly more than the layer of the fine ones, indicating that there is strong downslope velocity shear through the avalanche. The wavelength of the stripes is dominated by avalanching layer that is brought to rest by the shock.

A particularly interesting observation in figure $2(a)$ is that the thickness of the layer of large grains within the avalanche is approximately the same as the thickness of the freshly deposited layer at the base. This suggests that there is a regime in which all the large particles that reach the flow front are deposited on the underlying substrate and hence that the length scale of the coarse-particle-rich flow front is constant. Travelling-wave solutions in which the avalanche free surface, the basal deposition and the internal particle-size distribution are steady in a frame moving downslope 


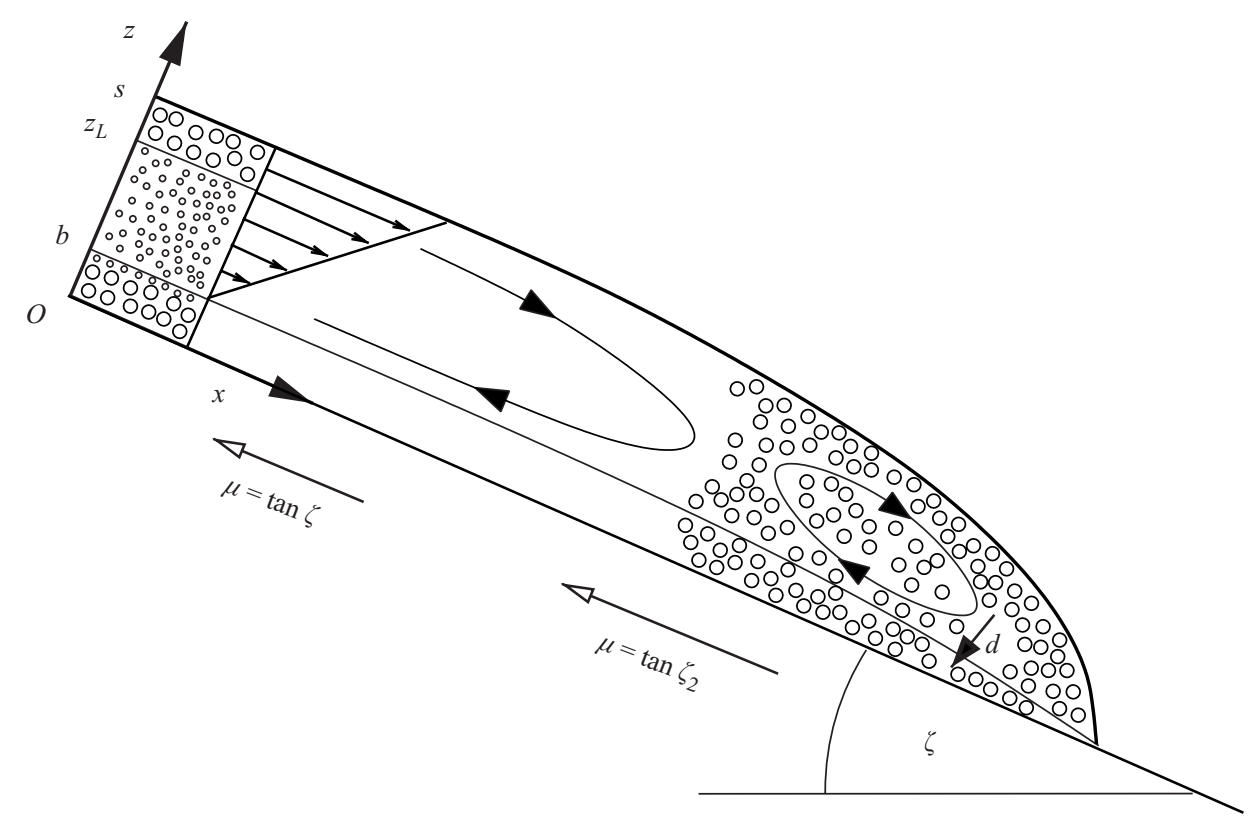

FIGURE 3. A schematic diagram showing the propagation of a granular avalanche down a rough slope inclined at an angle $\zeta$ to the horizontal. The $x$-axis is oriented in the downslope direction, and the $z$-axis is normal to it. The free surface lies at $z=s(x, t)$, and the base $z=b(x, t)$ may evolve due to deposition at a rate $d$ close to the flow front. Sufficiently far upstream the flow is steady and uniform with the friction $\mu=\tan \zeta$ balancing the gravitational acceleration. Close to the front the free surface steepens in order to overcome the increased resistance to motion, as $\mu=\tan \zeta_{2}>\tan \zeta$. There is a linear downslope velocity profile $u(x, z, t)$ through the avalanche depth $h$, which drives a bulk recirculation of the flow. Kinetic sieving and squeeze expulsion cause the large particles to rise towards the free surface, where the velocity is faster, and they are transported towards the flow front. Once they reach the margin, they are overrun and either are deposited or rise to the free surface again to create a recirculating loop. Far upstream the particle-size distribution can resemble a small-particle sandwich, with an inversely graded avalanche flowing on a normally graded deposit. For sharp segregation in a bidisperse mixture a sharp segregation shock is located at $z=z_{L}$.

with the speed of the front $u_{F}$, as shown in the schematic diagram in figure 3, can therefore be envisaged. In this paper exact solutions for this case are derived, which yield important insights into the structure of the particle-size distribution behind a coarse-particle-rich flow front and the concentration changes as particles are deposited at the base of the flow. There is also a very interesting analogy between the formation of two-dimensional stratification patterns and three-dimensional flows in which lateral levees are formed. In both cases, large rough grains rise to the surface of the flow by particle-size segregation and then are transported to the flow front by velocity shear, where they experience more resistance to motion and are deposited. The difference is that when the sidewalls are not there to suppress the lateral motion, the large rough grains prefer to be shouldered aside to form the lateral levees, instead of being deposited at the base and overrun.

\subsection{Kinetic sieving and squeeze expulsion}

At the heart of these processes is the segregation mechanism itself. Particles of different sizes, densities and roughnesses are notoriously prone to demixing during agitation and transport, and there may be competing mechanisms acting all at once. 
Broadly speaking, geologists have found that large clasts rise to the free surface of a flow through kinetic sieving and squeeze expulsion (Savage \& Lun 1988). These terms describe the processes in which small particles are statistically more likely to drop down through the gaps that open up beneath them and then lever large particles upwards. Other processes such as diffusive remixing (Hsiau \& Hunt 1993), convection (Erichs et al. 1995), differential fluid drag (Zhang \& Reese 2000), large inertia 'intruders' (Thomas 2000; Möbius et al. 2001) and polydispersity of the grain size population may complicate this simple picture, and it can be difficult to predict whether a particle will rise or fall relative to its neighbours. Nevertheless 'inverse' or 'reverse' grading (Middleton \& Hampton 1976) in which the entire grain population coarsens upwards is common in the field and widely attributed to vertical segregation within granular flows (e.g. Bagnold 1954).

Despite the importance of particle segregation in geophysical and industrial applications there have been surprisingly few attempts to model the process even for idealized bidisperse mixtures of grains. The first theory for dense granular avalanches was derived by Savage \& Lun (1988) from statistical mechanics and information entropy principles. Using a movable hopper, splitter plates and collection bins, Savage \& Lun (1988) and Vallance \& Savage (2000) were able to measure the steady-state two-dimensional particle-size distribution for a uniform concentration flow out of a hopper and show that it was in good agreement with predictions of the theory. There has also been work on dilute systems with models derived from kinetic theory (e.g. Jenkins \& Mancini 1987; Jenkins \& Yoon 2001) as well as heuristic arguments (Dolgunin \& Ukolov 1995). These theories include particle-size, particle-density and diffusive remixing and are more sophisticated than Savage \& Lun's (1988) model. However, the resulting equations are more complicated to solve, and so far only one-dimensional problems have been considered.

Recently a very simple description of particle-size segregation and diffusive remixing has been developed from two- and three-constituent mixture theory by Gray \& Thornton (2005), Gray \& Chugunov (2006) and Thornton et al. (2006). These models are related to earlier theories for segregation in bidisperse mixtures by Savage \& Lun (1988) and Dolgunin \& Ukolov (1995) and are capable of capturing the leading-order segregation effects in granular avalanches. The model assumes that the bulk flow is incompressible and has a lithostatic pressure distribution through its depth, so it is consistent with the assumptions of most depth-averaged avalanche models (e.g. Grigorian et al. 1967; Savage \& Hutter 1989; Iverson 1997; Gray et al. 1999; Pouliquen $1999 a, b$; Wieland et al. 1999; Denlinger \& Iverson 2001; Iverson \& Denlinger 2001; Gray et al. 2003; Cui et al. 2007; Gray \& Cui 2007). The hyperbolic version of the theory (Gray \& Thornton 2005; Thornton et al. 2006) has been used to construct two-dimensional steady-state and time-dependent exact solutions in uniform shear flows (Gray, Shearer \& Thornton 2006; Shearer, Gray \& Thornton 2008), which exhibit evolving concentration shocks, expansion fans and the formation of sharply segregated inversely graded layers. The parabolic theory (Gray \& Chugunov 2006) has an extra diffusion term that models the effects of diffusive remixing and smears out the sharp concentration shocks. It is harder to construct exact solutions for this case, although it is possible to use a linearizing transformation to obtain a general solution for one-dimensional time-dependent problems. Here the hyperbolic version of the theory is adopted for simplicity.

Of particular relevance to this paper is that it is possible to generate continuously breaking size-segregation waves (Thornton \& Gray 2008; McIntyre et al. 2008) that travel downstream at constant speed and allow the large and small particles to be 


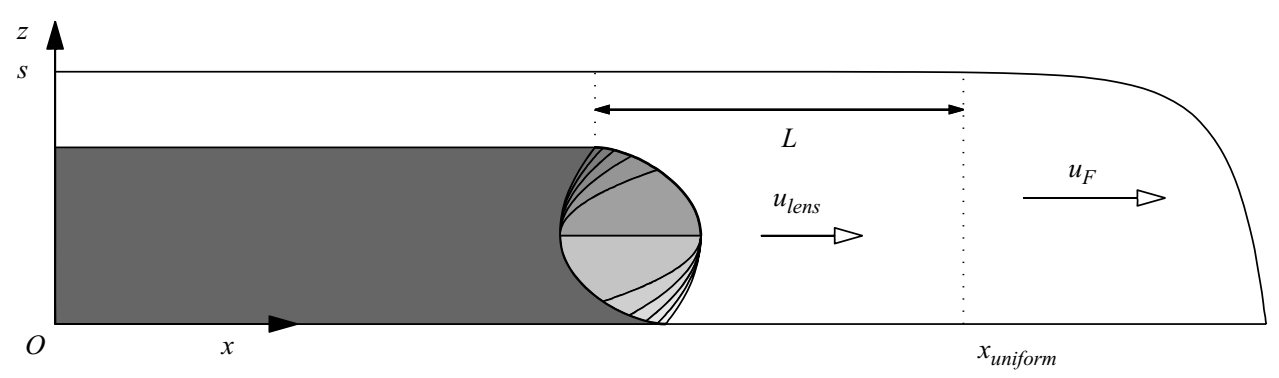

FIGURE 4. A vertical section through a steadily propagating avalanche showing the particle-size distribution in a growing coarse-particle front. Greyscale is used to indicate the particle concentration. The white regions corresponds to all large particles, dark grey to all fine ones and the lighter greys, at intervals of 0.1 units, to the concentrations in between. For sharp segregation in a uniform depth shear flow $\left(x \leqslant x_{\text {uniform }}\right)$ the coarse-particle front is connected to the inversely graded flow behind by a breaking size-segregation wave. This consists of two expansion fans and two concentration shocks arranged in a lens-like structure (Thornton \& Gray 2008) that propagates downslope with constant speed $u_{\text {lens }}$. This is equal to the depth-averaged velocity over its height and is necessarily less than the speed of the front $u_{F}$. The coarse front therefore grows linearly with increasing time, $L=L_{0}+\left(u_{F}-u_{\text {lens }}\right) t$.

recirculated in the flow. Figure 4 shows an extension of Thornton \& Gray's (2008) solution to the case of a coarse-particle-rich front travelling downslope with constant speed $u_{F}$. Sufficiently far upstream, the avalanche is of uniform thickness with a sharply segregated inversely graded concentration shock separating the large particles above from the fine ones below. These regions are joined by a 'lens-like' breaking size-segregation wave, which consists of two expansion fans and two concentration shocks that propagate downstream at a speed $u_{\text {lens }}$ equal to the depth-averaged velocity through the wave (Thornton \& Gray 2008). Since the lens does not extend through the entire flow depth, $u_{\text {lens }}$ is necessarily less than the front velocity $u_{F}$, and the coarse-particle-rich head grows linearly in time. The solution shown in figure 4 gives the first concrete structure for the particle-size distribution at an avalanche front and is applicable to uniform-thickness flows in which there is no deposition or lateral transport. In the case of stratification-pattern experiments shown in figure 1, basal deposition is vital to produce the small-particle sandwich in the deposit that was made visible in the composite image in figure 2. It is therefore of interest to solve the segregation equations in a non-uniform depositing flow.

\section{Reconstruction of the bulk motion near the flow front}

In $\S 1$ it was argued that the evolving particle-size distribution has a strong segregation-mobility feedback on the motion of the avalanche that causes the larger rougher particles to deposit on the underlying surface as they reach the flow front. The strongest evidence for this is the dramatic change between 'stratification' and 'segregation' patterns in heaps, as the relative roughness and size of the large and small particles are varied (Herrmann 1998). Given our relatively rudimentary understanding of particle-size segregation in non-uniform depositing flows, a fully coupled solution is beyond the scope of this paper. The primary aim here is to solve the segregation problem in a flow that captures the key qualitative features of the motion observed in the experiments in $\S 1.1$. In particular, a simplified depth-averaged travellingwave solution for the velocity $\overline{\boldsymbol{u}}$ will be constructed that has a well-defined steadily propagating flow front, near which grains are deposited, and far upstream the flow 
is steady and uniform with no further deposition. Assumed velocity profiles through the avalanche depth will then be used to reconstruct the bulk velocity field $\boldsymbol{u}$ needed by the segregation theory. Although this derivation does not solve the fully coupled problem, it illustrates some important aspects of how full coupling may be achieved. It also neglects sidewall friction which can have a controlling effect on the thickness and velocity of steady uniform flows that develop on top of the pile (Jop, Forterre \& Pouliquen 2005, 2006; Jesuthasan, Baliga \& Savage 2006; Savage 2008) with flows in a wide channel differing significantly from those observed in narrow channels.

Our premise in this paper is that the larger rougher grains are deposited near the flow front because of the greatly enhanced friction that they experience when they reach it; i.e. the deposition observed in stratification experiments is primarily a segregation-mobility feedback effect. Static layers may also be deposited in narrow channels because of sidewall friction (Taberlet et al. 2003, 2004). These flows are characterized by a steady uniform avalanche that forms on top of a super-stable heap of grains, which has a much higher angle of repose than one would usually expect. When the inflow stops, all the particles flow out, provided that the channel angle is sufficiently high. This is rather different from the deposition observed at low flow rates in the stratification experiments in $\S 1.1$, since both the initial and final pile surfaces are already raised to a slightly higher angle of repose by sidewall friction (Grasselli \& Herrmann 1997) and are stable even when no grains are flowing down them. The introduction of particles of different sizes and roughnesses introduces a rich variety of behaviour, which is rather different from what happens in steady monodisperse twodimensional granular flows down slopes or in very wide three-dimensional channels, where particle dynamics simulations (Silbert et al. 2001; Silbert, Landry \& Grest 2003; GDR MiDi 2004; Baran, Ertas \& Halsey 2006) and experiments (Deboeuf et al. 2006) show that no static layers develop. There is, however, no inherent contradiction between the results in the monodisperse and bidisperse systems.

\subsection{Governing equations}

Let $O x z$ be a coordinate system inclined at an angle $\zeta$ to the horizontal and aligned with the free surface of the static heap as shown in figure 3 . The $x$-axis is oriented in the downslope direction, and the $z$-axis is normal to the slope, with the free surface lying at a height $z=s(x, t)$ and the base at $z=b(x, t)$. It follows that the avalanche thickness measured normal to the slope is

$$
h(x, t)=s(x, t)-b(x, t) .
$$

The avalanche has velocity components $u(x, z, t)$ and $w(x, z, t)$ in the downslope and normal directions, respectively, and is assumed to be incompressible with a lithostatic pressure distribution through its depth. The depth-averaged downslope velocity is

$$
\bar{u}(x, t)=\frac{1}{h} \int_{b(x, t)}^{s(x, t)} u(x, z, t) \mathrm{d} z .
$$

Assuming that the flow is shallow the equations can be integrated normal to the slope to yield depth-averaged mass and momentum balance equations (Gray 2001) that include the effects of basal deposition,

$$
\begin{aligned}
\frac{\partial h}{\partial t}+\frac{\partial}{\partial x}(h \bar{u}) & =-d \\
\frac{\partial}{\partial t}(h \bar{u})+\frac{\partial}{\partial x}\left(F h \bar{u}^{2}\right)+\frac{\partial}{\partial x}\left(\frac{1}{2} g h^{2} \cos \zeta\right) & =h g \cos \zeta\left(\tan \zeta-\mu-\frac{\partial b}{\partial x}\right)-\bar{u} d
\end{aligned}
$$


where $d$ is deposition rate; $g$ is the coefficient of gravitational acceleration; $\mu$ is the basal friction coefficient; and the shape factor $F=\overline{u^{2}} / \bar{u}^{2}$ is assumed to be equal to unity for simplicity. These equations implicitly assume that the earth-pressure coefficient, used in some theories, is equal to unity and that the process of deposition has a neutral effect on the depth-averaged velocity. The non-strictly hyperbolic system ((2.3) and (2.4)) is closely related to the shallow-water equations of fluid mechanics and has wave speed $\sqrt{g h \cos \zeta}$. It follows that the Froude number can be defined as

$$
F r=\frac{\bar{u}}{\sqrt{g h \cos \zeta}} .
$$

The primary difference from shallow-water flows is the presence of source terms on the right-hand sides of (2.3) and (2.4). These represent the physical effects of gravity acceleration, basal friction, changes in topography and deposition of mass. Finally, it is useful to supplement these equations with the kinematic condition for the evolution of the basal surface $b$ as mass is deposited. This states that on

$$
z=b(x, t), \quad \frac{\partial b}{\partial t}+u_{b} \frac{\partial b}{\partial x}-w_{b}=d,
$$

where $u_{b}$ and $w_{b}$ are the velocity components at the base of the avalanche.

\subsection{Travelling waves with erosion and deposition}

Motivated by the observations of the flow fronts in the stratification experiments in $\S 1.1$, a travelling-wave solution is sought for a front propagating downslope with speed $u_{F}$. Introducing a front-centred coordinate system $(\xi, \tau)$,

$$
\xi=x-u_{F} t, \quad \tau=t,
$$

(2.3), (2.4) and (2.6) transform into

$$
\begin{aligned}
\frac{\partial}{\partial \xi}\left(h\left(\bar{u}-u_{F}\right)\right) & =-d, \\
\left(\bar{u}-u_{F}\right) \frac{\partial \bar{u}}{\partial \xi}+g \cos \zeta \frac{\partial s}{\partial \xi} & =g \cos \zeta(\tan \zeta-\mu), \\
\left(u_{b}-u_{F}\right) \frac{\partial b}{\partial \xi}-w_{b} & =d,
\end{aligned}
$$

where the depth-averaged momentum balance equation (2.9) has been simplified with the use of (2.8), and the topography and pressure gradient terms have been combined to yield a gradient in the free-surface height $s$.

Assuming that the velocity at the base of the avalanche is zero, i.e. $u_{b}=0$ and $w_{b}=0,(2.10)$ implies that the deposition rate $d=-u_{F} \partial b / \partial \xi$. This allows the depthaveraged mass balance equation (2.8) to be integrated subject to the boundary condition $h=b=0$ at $\xi=0$, to show that

$$
b=\lambda h,
$$

where

$$
\lambda=\frac{\bar{u}-u_{F}}{u_{F}}
$$

is the non-dimensional velocity difference between the bulk flow and the front. Equation (2.11) relates the basal topography height $b$ to the avalanche thickness $h$, the depth-averaged velocity $\bar{u}$ and the front speed $u_{F}$. Equation (2.9) provides the 
only other independent equation to calculate one of the remaining unknowns, and it is necessary to make another assumption to close the problem. One approach, which has been used successfully by Doyle et al. (2007) to model collapsing column experiments (Lajeunesse, Mangeney-Castelnau \& Vilotte 2004; Lube et al. 2004; Balmforth \& Kerswell 2005) is to prescribe the deposition rate $d$. Here, however, Pouliquen's (1999b) original hypothesis that the mean velocity is equal to a constant throughout the avalanche is followed, i.e.

$$
\bar{u}=(1+\lambda) u_{F}, \quad \forall \xi
$$

where $\lambda$ becomes a constant parameter that determines the amount of deposition. Note this is a model assumption when $\lambda \neq 0$, but when there in no deposition $(\lambda=0)$ it is imposed by the depth-averaged mass balance equation (2.8). In the solution presented here, the deposition is uncoupled from the evolving particle-size distribution and is prescribed by setting the value of $\lambda$. However, the overall premise of this paper is that larger rougher particles that reach the flow front are deposited because of the enhanced frictional resistance to motion that they experience there. It follows that in a fully coupled theory the deposition rate $d$ would be coupled with the evolving size distribution and the dynamics of the avalanche front.

Since there are no gradients in $\bar{u}$ the depth-averaged momentum balance equation (2.9) reduces to

$$
\frac{\partial s}{\partial \xi}=\tan \zeta-\mu,
$$

which is identical to equation (3) in Pouliquen (1999b) in the absence of deposition. It describes a simple balance between the free-surface gradient, gravitational acceleration and basal friction. This is one of the points in the theory where additional effects could be added to make the bulk flow more realistic. For instance, a fully coupled model can be created by treating the basal friction $\mu$ as a volume fraction weighted average of the friction associated with each of the pure phases (e.g. Pouliquen \& Vallance 1999), and wall friction effects (e.g. Taberlet et al. 2003, 2004; Jop et al. 2005, 2006; Jesuthasan et al. 2006; Savage 2008) could also be added. However, since so little is known about the nature of the solutions to the particle-size segregation problem, an uncoupled empirical law for flows down rough inclined planes (Pouliquen \& Forterre 2002) will be used at this stage. The Pouliquen \& Forterre (2002) friction law assumes that for inclination angles below $\zeta_{1}$ there is no flow; for angles in the range $\zeta_{1}<\zeta<\zeta_{2}$ steady uniform flows develop; and for inclinations above $\zeta_{2}$ the flow is accelerated. It is dependent on the local thickness and Froude number of the flow,

$$
\mu(h, F r)=\tan \zeta_{1}+\frac{\tan \zeta_{2}-\tan \zeta_{1}}{1+\beta h /(\mathscr{L} F r)},
$$

where the empirical constant $\beta=0.136$ and $\mathscr{L}$ is typical length scale for the transition between $\zeta_{1}$ and $\zeta_{2}$. It has the advantage over the exponential form of the friction law (Pouliquen 1999a,b) in that it allows an exact solution for the free-surface shape to be constructed. Defining the parameter

$$
\gamma=\frac{\tan \zeta_{2}-\tan \zeta_{1}}{\tan \zeta-\tan \zeta_{1}}-1
$$

and considering what happens in the far field, where $\partial s / \partial \xi=0$ and where the flow depth is equal to $h_{\infty}$, it follows from (2.5) and (2.14)-(2.16) that the depth-averaged 


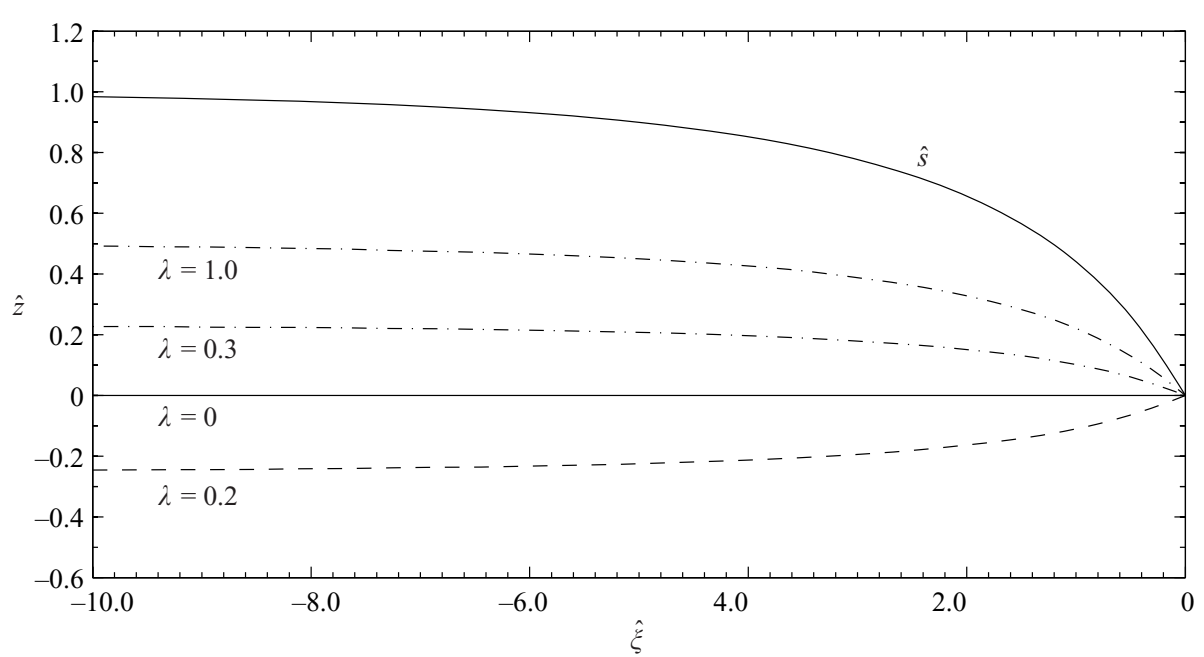

FIGURE 5. The shape of the avalanche free surface $\hat{s}$ is plotted as a function of $\hat{\xi}$ using a solid line for $\gamma=1.5$. It is independent of the deposition. The basal topography height $\hat{b}$ is also plotted as a function of $\hat{\xi}$ for deposition parameter $\lambda=1,0.3,0,-0.2$. The dot-dashed curves correspond to depositing flows, the dashed curve to erosive flows and the solid straight line to a fixed bed.

velocity

$$
\bar{u}=\left(\frac{\beta \sqrt{g \cos \zeta}}{\mathscr{L} \gamma}\right) h_{\infty}^{3 / 2}
$$

This has the well-known thickness to three halves velocity scaling (Vallance 1994; Azanza 1998; Pouliquen 1999a) and is, by assumption, constant throughout the flow. The front speed $u_{F}$ can then be calculated by (2.13). It is convenient to introduce non-dimensional coordinates

$$
z=s_{\infty} \hat{z}, \quad \xi=\frac{s_{\infty}}{\tan \zeta_{2}-\tan \zeta_{1}} \hat{\xi},
$$

where $s_{\infty}=(1+\lambda) h_{\infty}$ is the far field free-surface height. Using (2.17) to eliminate $\bar{u}$ from $(2.15)$ and the identity $\hat{s}=(1+\lambda) \hat{h},(2.14)$ reduces to the separable ordinary differential equation

$$
\frac{\mathrm{d} \hat{s}}{\mathrm{~d} \hat{\xi}}=\frac{1}{1+\gamma}-\frac{1}{1+\gamma \hat{s}^{3 / 2}} .
$$

This degenerates to $\mathrm{d} \hat{s} / \mathrm{d} \hat{\xi}=0$ when $\gamma=0$ and as $\gamma \rightarrow \infty$, which if $\hat{s}(0)=0$ implies that $\hat{s}=0$ everywhere. For finite non-zero $\gamma,(2.19)$ may be integrated exactly subject to $\hat{s}(0)=0$, to give the position $\hat{\xi}$ in terms of the thickness $\hat{s} \in[0,1]$ :

$$
\hat{\xi}=(1+\gamma) \hat{s}+\frac{(1+\gamma)^{2}}{3 \gamma}\left[\ln \left(\frac{(1-\sqrt{\hat{s}})^{2}}{\hat{s}+\sqrt{\hat{s}}+1}\right)+2 \sqrt{3} \operatorname{atan}\left(\frac{1+2 \sqrt{\hat{s}}}{\sqrt{3}}\right)-\frac{\pi}{\sqrt{3}}\right] .
$$

The solution is independent of the deposition parameter $\lambda$ and is shown in figure 5 for $\gamma=1.5$. The front lies at $\hat{\xi}=0$ and approaches unity as $\hat{\xi} \rightarrow-\infty$. The characteristic convex shape arises because the basal friction coefficient $\mu$ increases from $\tan \zeta$ to $\tan \zeta_{2}$ as the flow thickness decreases from $h_{\infty}$ to 0 in (2.15), which must be balanced by a negative free-surface gradient in (2.14). 
The deposit height $b$ can be non-dimensionalized by (2.18) and expressed in terms of the free-surface height $\hat{s}$ by (2.1), to give

$$
\hat{b}=\frac{\lambda}{1+\lambda} \hat{s}
$$

Deposit profiles are illustrated in figure 5 for $\lambda=1,0.3,0$ and -0.2 and $\gamma=1.5$. In all cases the majority of the deposition/erosion occurs close to the flow front where the free-surface gradients are highest, consistent with the experiments in $\S 1.1$. For depositing flows in which $\lambda>0$ the depth-averaged velocity $\bar{u}$ is greater than $u_{F}$, by (2.13), implying that there is a net transport of grains towards the front. However, it is also possible to construct steadily propagating erosive fronts with $\lambda<0$, and these may be useful for describing the metastable experiments of Daerr (2001), Ancey (2002) and Douady et al. (2002), as well as erosive fronts in snow avalanches (Issler 2003).

\subsection{Reconstruction of the two-dimensional bulk flow field}

A significant contribution of this paper is to show how the two-dimensional bulk flow field can be reconstructed from a one-dimensional depth-averaged avalanche solution, using assumed velocity profiles through the avalanche depth. For simplicity the downslope velocity is assumed to be linear and satisfies the no-slip condition at the base:

$$
u= \begin{cases}2 \bar{u}\left(\frac{z-b}{h}\right), & b \leqslant z \leqslant s, \\ 0, & 0 \leqslant z<b,\end{cases}
$$

where the depth-averaged velocity $\bar{u}$ is constant by (2.13). There is nothing inherently special about a linear velocity profile, and most of the results generalize easily to nonlinear velocity profiles (e.g. Thornton \& Gray 2008). The shape of the assumed velocity profile provides a further point at which a more general fully coupled theory could take account of the local particle-size distribution. Indeed, there are now discrete numerical simulations of bidisperse disks (Linares-Guerrero et al. 2007; Rognon et al. 2007) and experiments (Phillips et al. 2006) that suggest that the feedback on the flow profiles may be important in enhancing the run-out of bidisperse avalanches.

The normal velocity $w$ can be reconstructed by integrating the incompressibility condition,

$$
\frac{\partial u}{\partial \xi}+\frac{\partial w}{\partial z}=0
$$

through the avalanche depth, subject to the condition that $w=0$ on $z=b$. Using (2.11) and the fact that $\bar{u}$ and $\lambda$ are constants, the normal velocity throughout the avalanche is

$$
w= \begin{cases}\frac{\bar{u}}{h^{2}} \frac{\mathrm{d} h}{\mathrm{~d} \xi}\left(z^{2}-b^{2}\right), & b \leqslant z \leqslant s, \\ 0, & 0 \leqslant z<b .\end{cases}
$$

In the moving coordinate system the particle path $z=z_{p}(\xi)$ is given by

$$
\frac{\mathrm{d} \xi}{\mathrm{d} \tau}=u-u_{F}, \quad \frac{\mathrm{d} z_{p}}{\mathrm{~d} \tau}=w .
$$

Since the velocity field is steady, time may be eliminated to give

$$
\frac{\mathrm{d} z_{p}}{\mathrm{~d} \xi}=\frac{w}{u-u_{F}} \text {. }
$$


Following Gray \& Thornton (2005) and Thornton \& Gray (2008), a depth-integrated velocity coordinate

$$
\psi=\int_{0}^{z} u\left(\xi, z^{\prime}\right)-u_{F} \mathrm{~d} z^{\prime}
$$

is introduced to transform the normal coordinate $z$. If $\psi_{p}(\xi)$ is the particle path $z=z_{p}(\xi)$, expressed in depth-integrated velocity coordinates, then differentiating (2.27) with respect to $\xi$, using Leibniz's rule (Abramowitz \& Stegun 1970) and the incompressibility condition (2.23), yields the relation

$$
\frac{\mathrm{d} \psi_{p}}{\mathrm{~d} \xi}=\left(u-u_{F}\right) \frac{\mathrm{d} z_{p}}{\mathrm{~d} \xi}-w .
$$

The particle path equation (2.26) in depth-integrated velocity coordinates therefore reduces to the trivial ordinary differential equation

$$
\frac{\mathrm{d} \psi_{p}}{\mathrm{~d} \xi}=0 \quad \Rightarrow \quad \psi_{p}=\psi_{\infty} .
$$

That is each particle path $\psi_{p}$ is equal to a constant value $\psi_{\infty}$ determined at the inflow as $\xi \rightarrow-\infty$. For the linear velocity field the coordinate $\psi$ can be calculated explicitly by substituting (2.22) into (2.27) and integrating to give

$$
\psi=\left\{\begin{array}{cc}
\bar{u}\left(z^{2}-\kappa z h+\lambda^{2} h^{2}\right), & b \leqslant z \leqslant s, \\
-u_{F} z, & 0 \leqslant z<b,
\end{array}\right.
$$

where $\kappa$ is a function of the deposition parameter $\lambda$ :

$$
\kappa=2 \lambda+\frac{1}{1+\lambda} .
$$

The depth-integrated velocity coordinate $\psi$ is in fact the streamfunction, since

$$
\frac{\partial \psi}{\partial z}=u-u_{F}, \quad \frac{\partial \psi}{\partial \xi}=-w, \quad 0 \leqslant z \leqslant s,
$$

and therefore automatically satisfies the incompressibility condition (2.23). The streamfunction coordinates $(\xi, \psi)$ are very useful for constructing exact solutions to the segregation equation in $\S 3$, and it is important to understand some of their properties. The streamfunction $\psi$ is zero along the avalanche free surface $z=s$ and the chute surface $z=0$. For fixed $h$, it decreases to a local minimum at $z_{u_{F}}=\kappa h / 2$ and has a global minimum at $z_{u_{F}}^{\infty}=\kappa h_{\infty} / 2$ as $\xi \rightarrow-\infty$. Introducing non-dimensional variables

$$
u=u_{F} \hat{u}, \quad w=u_{F}\left(\tan \zeta_{2}-\tan \zeta_{1}\right) \hat{w}, \quad \psi=s_{\infty} u_{F} \hat{\psi},
$$

which reflect the shallowness of the spatial non-dimensionalization in (2.18), the nondimensional streamfunction becomes

$$
\hat{\psi}=\left\{\begin{array}{cc}
\frac{1+\lambda}{\hat{h}}\left(\hat{z}^{2}-\kappa \hat{z} \hat{h}+\lambda^{2} \hat{h}^{2}\right), & \hat{b} \leqslant \hat{z} \leqslant \hat{s}, \\
-\hat{z}, & 0 \leqslant \hat{z}<\hat{b} .
\end{array}\right.
$$

In streamfunction coordinates, the no-mean-flow line $\hat{z}_{u_{F}}=\kappa \hat{h} / 2$ is

$$
\hat{\psi}_{u_{F}}=\left(\lambda^{2}-\kappa^{2} / 4\right) \hat{s},
$$




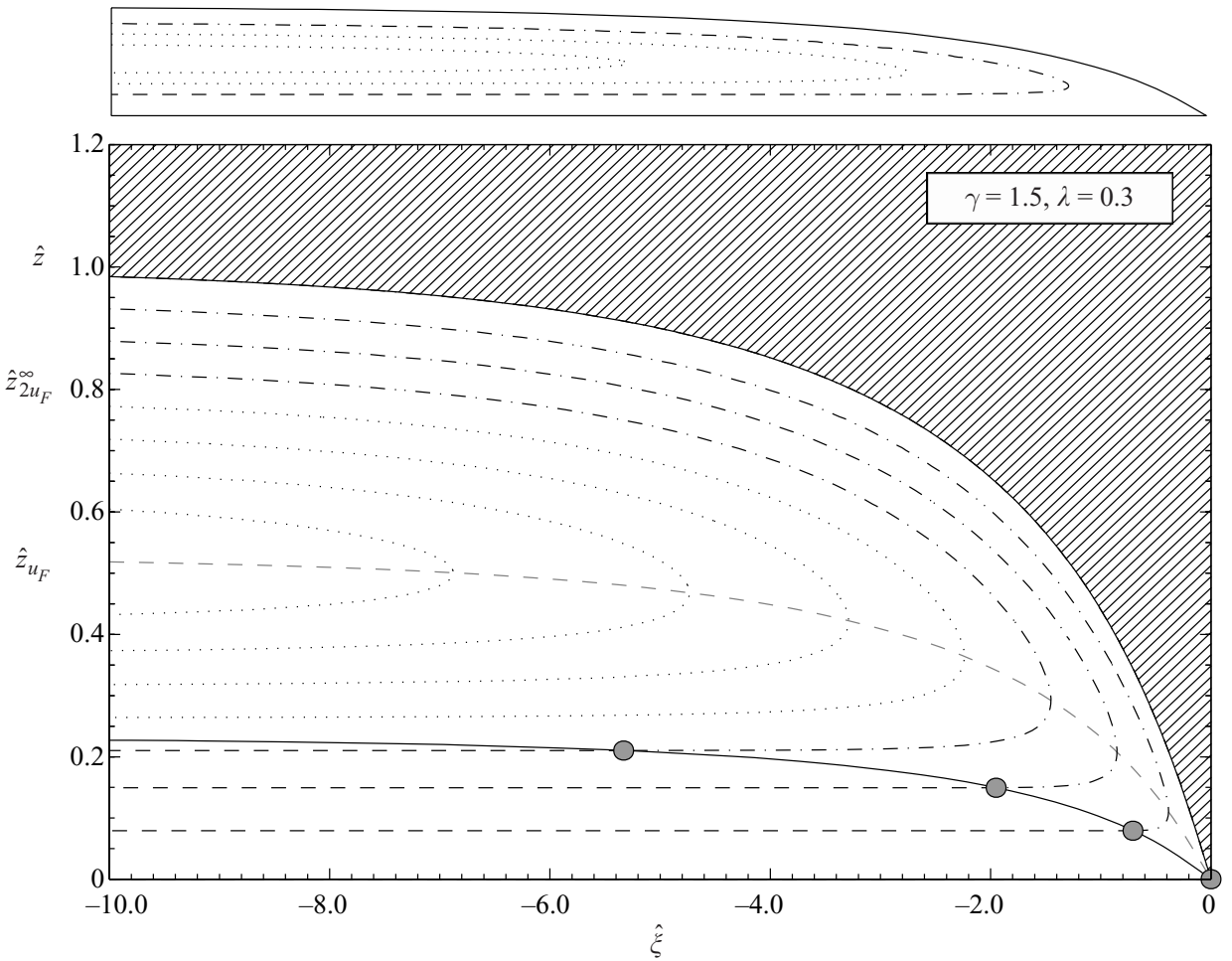

FiguRE 6. The particle paths at a depositional flow front given by parameters $\gamma=1.5$ and $\lambda=0.3$. The free surface and basal deposition interface are shown with solid black lines, and the hatched region is empty. The material above the no-mean-flow line $\hat{z}_{u_{F}}$ (grey dashed line) moves towards the front, and that below it move backwards. Particles starting below $\hat{z}_{2 u_{F}}^{\infty}$ far upstream are recirculated (dotted lines), while those starting above $\hat{z}_{2 u_{F}}^{\infty}$ (dot-dashed curves) are deposited (grey circles) and move back along the black dashed lines. Above the main figure is a compressed image showing the solution in a more realistic aspect ratio.

and the basal deposition surface $\hat{b}$ can be expressed as

$$
\hat{\psi}_{b}=-\frac{\lambda}{1+\lambda} \hat{s},
$$

by (2.21) and (2.34). These are shown in figure 6. The no-mean-flow line $\hat{\psi}_{u_{F}}$ marks the local minimum of $\hat{\psi}$ for fixed $\hat{s}$ and divides the $\hat{\psi}$ coordinate system into upper and lower domains. In the upper domain the bulk flow is from left to right, towards the front, While in the lower domain, the flow is from right to left, away from the front. The non-dimensional height of a point $(\hat{\xi}, \hat{\psi})$ can be found by iterating for $\hat{s} \in[0,1]$ using (2.20), computing the equivalent avalanche thickness $\hat{h}=\hat{s} /(1+\lambda)$ and then using

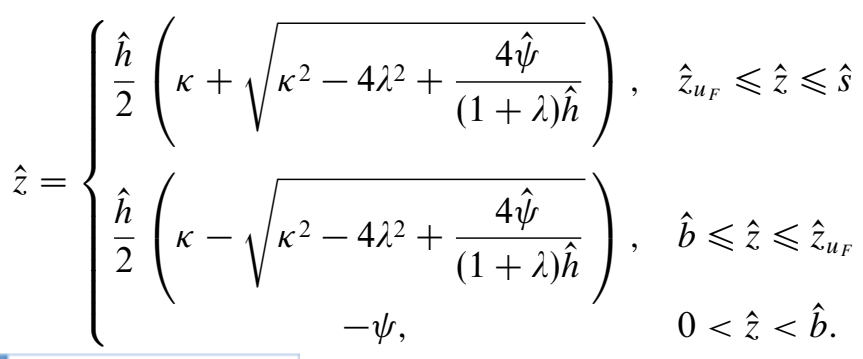


Since there are two values of $\hat{z}$ for each value of $\hat{\psi}$, it is important to keep track of which domain the solution is in, when mapping it back into physical space.

If a particle enters the avalanche at a height $\hat{z}_{i n}$ as $\hat{\xi} \rightarrow-\infty$, and the avalanche thickness in the far field is $\hat{h}_{\infty}$, then, since $\hat{\psi}$ is constant along a particle path, (2.34) implies that the particle will exit at height

$$
\hat{z}_{\text {exit }}=\kappa \hat{h}_{\infty}-\hat{z}_{\text {in }}
$$

as $\hat{\xi} \rightarrow-\infty$ again, provided that it stays within the flowing layer. Equation (2.38) allows us to determine some important limiting values. Firstly, the inflow and exit heights are the same at $\hat{z}_{u_{F}}^{\infty}=\kappa \hat{h}_{\infty} / 2$. Moreover, the exit height is equal to the deposit height $\hat{b}_{\infty}$ in the far field if $\hat{z}_{i n}$ is equal to $\hat{z}_{2 u_{F}}^{\infty}=(\kappa-\lambda) \hat{h}_{\infty}$, which is the height at which the velocity is equal to twice the front speed, $\hat{u}_{\infty}=2 \hat{u}_{F}$, as $\hat{\xi} \rightarrow-\infty$. Particles are therefore recirculated within the flow, provided $\hat{z}_{u_{F}}^{\infty}<\hat{z}_{i n}<\hat{z}_{2 u_{F}}^{\infty}$. Each flow path may be parameterized by defining the height $\hat{z}_{p} \in\left[\hat{z}_{i n}, \hat{z}_{\text {exit }}\right]$ and solving the quadratic equation (2.34) for the associated avalanche thickness,

$$
\hat{h}_{p}=\frac{\left[(1+\lambda) \kappa \hat{z}_{p}+\hat{\psi}_{\infty}\right]-\sqrt{\left[(1+\lambda) \kappa \hat{z}_{p}+\hat{\psi}_{\infty}\right]^{2}-4(1+\lambda)^{2} \lambda^{2} \hat{z}_{p}^{2}}}{2(1+\lambda) \lambda^{2}},
$$

where $\hat{\psi}_{\infty}$ is determined by substituting $\hat{z}_{\text {in }}$ and $\hat{h}_{\infty}$ into (2.34). From (2.1), (2.11) and (2.18), the corresponding free-surface height

$$
\hat{s}_{p}=(1+\lambda) \hat{h}_{p}
$$

and from (2.20) its spatial position is given explicitly by

$$
\hat{\xi}_{p}=(1+\gamma) \hat{s}_{p}+\frac{(1+\gamma)^{2}}{3 \gamma}\left[\ln \left(\frac{\left(1-\sqrt{\hat{s}_{p}}\right)^{2}}{\hat{s}_{p}+\sqrt{\hat{s}_{p}}+1}\right)+2 \sqrt{3} \operatorname{atan}\left(\frac{1+2 \sqrt{\hat{s}_{p}}}{\sqrt{3}}\right)-\frac{\pi}{\sqrt{3}}\right] .
$$

The recirculating flow paths are shown as dotted lines in figure 6. Particles enter from the left in $\left[\hat{z}_{u_{F}}^{\infty}, \hat{z}_{2 u_{F}}^{\infty}\right]$ and are transported around in a series of arcs that exit in $\left[\lambda \hat{h}_{\infty}, \hat{z}_{u_{F}}^{\infty}\right]$. Equation (2.26) implies that each arc has an infinite gradient as it crosses the no-net-transport line $\hat{z}_{u_{F}}=\kappa \hat{h} / 2$, which is where $\hat{u}=\hat{u}_{F}$. Particle paths that start at a height $\hat{z}_{i n}>\hat{z}_{2 u_{F}}^{\infty}$ are deposited during the flow, and their deposit height $\hat{z}_{\text {dep }}$ can be determined by setting $\hat{h}=\hat{z} / \lambda$ in (2.34) to show that

$$
\hat{z}_{\text {dep }}=-\hat{\psi}_{\infty} \text {. }
$$

The particle paths in the flowing region can therefore be parameterized by $\hat{z}_{p} \in$ $\left[\hat{z}_{i n}, \hat{z}_{\text {dep }}\right]$ and using (2.39)-(2.41) to determine the corresponding position $\hat{\xi}_{p}$ as before. These paths are shown as dot-dashed curves in figure 6. Once the particles are deposited they move upstream relative to the moving front, and (2.34) implies that they stay at their initial deposition height, $\hat{z}_{\text {dep }}$, given by (2.42). These correspond to the straight dashed lines in figure 6 .

\section{Particle-size segregation at a depositional flow front}

A very simple approach to modelling particle-size segregation in granular avalanches is adopted (Gray \& Thornton 2005; Thornton et al. 2006), which assumes that there is an idealized bidisperse distribution of large and small particles, that there 
is no diffusive remixing and that there are no density effects. In this case the theory reduces to a single scaler hyperbolic equation for the volume fraction of small particles $\phi \in[0,1]$ per unit granular volume. The volume fraction of large particles per unit granular volume is $1-\phi$, and the solids volume fraction $v$ is assumed to be uniform throughout the avalanche. Particle-size segregation in granular avalanches is driven by a combination of kinetic sieving and squeeze expulsion, and their net effect is accounted for by considering the momentum balance components of each constituent normal to the slope. The model rests on the observation that the large particles support more of the overburden pressure, as the small particles percolate through the interstices, but once segregation has ceased they must both support the overlying grains. Assuming that normal accelerations are small, the normal components of the large- and small-particle non-dimensional velocities are

$$
\begin{aligned}
\hat{w}^{l} & =\hat{w}+S_{r} \phi, \\
\hat{w}^{s} & =\hat{w}-S_{r}(1-\phi),
\end{aligned}
$$

where the subscripts ' $l$ ' and ' $s$ ' are for 'large' and 'small', respectively. The nondimensional segregation number

$$
S_{r}=\frac{q}{u_{F}\left(\tan \zeta_{2}-\tan \zeta_{1}\right)}
$$

implied by the scalings (2.18) and (2.33), is the ratio of a typical time scale for downstream transport $s_{\infty} /\left(u_{F}\left(\tan \zeta_{2}-\tan \zeta_{1}\right)\right)$ to a typical time scale for segregation $s_{\infty} / q$, where $q$ is the mean segregation velocity. These equations imply that the large particles will go up until there are no more fine ones to go down and that the small particles will go down until there are no more big particles to go up. The downstream velocity is assumed to be unaffected by the segregation, i.e. $\hat{u}^{l}=\hat{u}^{s}=\hat{u}$. When (3.2) is substituted into the small-particle mass balance equation (e.g. see Gray \& Thornton 2005, equation (2.2)) it yields the conservative form of the hyperbolic segregation equation

$$
\frac{\partial \phi}{\partial \hat{t}}+\frac{\partial}{\partial \hat{x}}(\phi \hat{u})+\frac{\partial}{\partial \hat{z}}(\phi \hat{w})-\frac{\partial}{\partial \hat{z}}\left(S_{r} \phi(1-\phi)\right)=0 .
$$

Using the bulk incompressibility equation (2.23) and assuming $S_{r}$ is constant in the flowing avalanche, (3.4) reduces to

$$
\frac{\partial \phi}{\partial \hat{t}}+\hat{u} \frac{\partial \phi}{\partial \hat{x}}+\hat{w} \frac{\partial \phi}{\partial \hat{z}}-S_{r} \frac{\partial}{\partial \hat{z}}(\phi(1-\phi))=0 .
$$

The final term on the left-hand side accounts for the effects of segregation. When it is zero, (3.5) reduces to an equation for a 'passive' tracer, and the small-particle concentration $\phi$ is simply swept along with the bulk flow. Segregation shuts off when $\phi$ equals either zero or unity, so there is no further sorting once either the large or the small particles have separated out into a pure phase. The concentration $\phi$ therefore automatically stays in the range $[0,1]$.

\subsection{Characteristics, jump conditions and particle paths}

The stratification experiments shown in figures 1 and 2 suggest that there are depositional solutions in which the the length of the coarse-particle-rich flow front remains constant. In order to investigate such solutions, it is convenient to introduce a change of variables,

$$
\hat{\tau}=\hat{t}, \quad \hat{\xi}=\hat{x}-\hat{u}_{F} \hat{t}, \quad \hat{\psi}=\int_{0}^{\hat{z}} \hat{u}\left(\hat{\xi}, \hat{z}^{\prime}\right)-\hat{u}_{F} \mathrm{~d} \hat{z}^{\prime},
$$


to transform (3.5) into a frame moving downslope at the front speed $\hat{u}_{F}=1$ and to map the $\hat{z}$-coordinate into the streamfunction coordinate $\hat{\psi}$, described in $\S 2.3$. For steady solutions in the travelling frame, $\partial \phi / \partial \hat{\tau}=0$, which allows a factor $\hat{u}-\hat{u}_{F}$ to be cancelled, to leave a quasi-linear equation for the small-particle concentration $\phi$ :

$$
\frac{\partial \phi}{\partial \hat{\xi}}-S_{r} \frac{\partial}{\partial \hat{\psi}}(\phi(1-\phi))=0 .
$$

This can be solved by the method of characteristics. For a characteristic starting at $\left(\hat{\xi}_{o}, \hat{\psi}_{o}\right)$ the concentration is equal to the constant $\phi_{o}$ along the line

$$
\hat{\psi}=\hat{\psi}_{o}+S_{r}\left(2 \phi_{o}-1\right)\left(\hat{\xi}-\hat{\xi}_{o}\right) \text {. }
$$

When the characteristics intersect discontinuous concentration shocks are formed. A limiting argument (e.g. Chadwick 1999) can be used to derive the concentration jump condition from (3.4), which holds at the discontinuity. If $\hat{z}=\hat{z}(\hat{x}, \hat{t})$ is the shock surface, propagating with speed $\hat{v}_{n}$ in the direction of the unit normal $\hat{\boldsymbol{n}}$, then the shock must satisfy

$$
\llbracket \phi\left(\hat{\boldsymbol{u}} \cdot \hat{\boldsymbol{n}}-\hat{v}_{n}\right) \rrbracket=\llbracket S_{r} \phi(1-\phi) \boldsymbol{k} \cdot \hat{\boldsymbol{n}} \rrbracket,
$$

where the jump bracket $\llbracket f \rrbracket=f^{+}-f^{-}$is the difference of the enclosed quantity on the forward ' + ' and rearward ' - ' sides of the discontinuity, and $\boldsymbol{k}$ is the unit vector normal to the slope. For the case of a steady travelling wave in the frame moving with the front, the velocity $\hat{\boldsymbol{u}}=\left(\hat{u}-\hat{u}_{F}, \hat{w}\right)$, the unit normal $\hat{\boldsymbol{n}}=(-\mathrm{d} \hat{z} / \mathrm{d} \hat{\xi}, 1) /\left(1+(\mathrm{d} \hat{z} / \mathrm{d} \hat{\xi})^{2}\right)^{1 / 2}$ and the normal propagation speed $\hat{v}_{n}=0$, which implies that the shock condition is

$$
\left(\hat{u}-\hat{u}_{F}\right) \frac{\mathrm{d} \hat{z}}{\mathrm{~d} \hat{\xi}}-\hat{w}=S_{r}\left(\phi^{+}+\phi^{-}-1\right) .
$$

By switching to the streamfunction coordinates (2.27) this reduces to

$$
\frac{\mathrm{d} \hat{\psi}}{\mathrm{d} \hat{\xi}}=S_{r}\left(\phi^{+}+\phi^{-}-1\right) .
$$

There is an interesting correspondence between the shock condition (3.11) and the paths of the large and small particles. The velocity components defined in (3.1) and (3.2) imply that these satisfy the three equations

$$
\frac{\mathrm{d} \hat{\xi}}{\mathrm{d} \hat{\tau}}=\hat{u}-\hat{u}_{F}, \quad \frac{\mathrm{d} \hat{z}^{l}}{\mathrm{~d} \hat{\tau}}=\hat{w}+S_{r} \phi, \quad \frac{\mathrm{d} \hat{z}^{s}}{\mathrm{~d} \hat{\tau}}=\hat{w}-S_{r}(1-\phi) .
$$

Since the velocities are independent of time

$$
\frac{\mathrm{d} \hat{z}^{l}}{\mathrm{~d} \hat{\xi}}=\frac{\hat{w}+S_{r} \phi}{\hat{u}-\hat{u}_{F}}, \quad \frac{\mathrm{d} \hat{z}^{s}}{\mathrm{~d} \hat{\xi}}=\frac{\hat{w}-S_{r}(1-\phi)}{\hat{u}-\hat{u}_{F}},
$$

which on transformation into streamfunction coordinates implies that the large- and small-particle paths are governed by

$$
\begin{aligned}
\frac{\mathrm{d} \hat{\psi}^{l}}{\mathrm{~d} \hat{\xi}} & =S_{r} \phi, \\
\frac{\mathrm{d} \hat{\psi}^{s}}{\mathrm{~d} \hat{\xi}} & =-S_{r}(1-\phi),
\end{aligned}
$$

respectively. The equation for the large-particle path (3.14) is identical to the equation for a shock (3.11) when $\phi^{+}=1$, while the small-particle path equation (3.15) is 
equivalent to the shock equation (3.11) when $\phi^{+}=0$. Tracking large particles is therefore equivalent to solving for a shock with small particles on the forward side, and tracking small particles is equivalent to solving for a shock with large particles on the other side. This cross-correspondence proves useful later on, as the solution involves a particle path that turns into a shock at an unknown location.

\subsection{Structure of the breaking size-segregation wave at the flow front}

In the far field the flowing part of the avalanche is assumed to be inversely graded, so that all the large particles lie above the small ones. Putting $\phi^{+}=0$ and $\phi^{-}=1$ in the shock condition (3.11) yields the trivial ordinary differential equation $\mathrm{d} \hat{\psi} / \mathrm{d} \hat{\xi}=0$. The incoming concentration jump therefore lies along the particle path $\hat{\psi}=\hat{\psi}_{L}$ in the upper domain. It is immediately apparent that if there are more large particles being transported towards the flow front than can be deposited into the stationary layer, then the front will grow with increasing time, as in figure 4. A necessary condition for breaking size-segregation waves, which travel at the same speed as the front $\hat{u}_{F}$, is that

$$
\hat{z}_{L}^{\infty}>\hat{z}_{2 u_{F}}^{\infty},
$$

where $\hat{z}_{L}^{\infty}$ is the height of the $\hat{\psi}_{L}$ path as $\hat{\xi} \rightarrow-\infty$, and the height $\hat{z}_{2 u_{F}}^{\infty}$ marks the transition between recirculating (dotted) and depositing (dot-dashed/dashed) bulkparticle paths in figure 6. The inequality (3.16) imposes an important restriction on the composition of the mixture and the total amount of deposition in the flow. The global concentration of small particles $\Phi$ can be calculated by integrating the mass flux of the fine ones $\phi \hat{u}$ between $\hat{b}$ and $\hat{s}$ at the inflow and dividing by the bulk mass flux $\hat{h} \hat{\bar{u}}$. Using (2.21) and (2.31) and the inequality (3.16) it follows that $\Phi$ satisfies the inequality

$$
\Phi=\frac{1}{\hat{h} \hat{\bar{u}}} \int_{\hat{b}_{\infty}}^{\hat{s}_{\infty}} \phi_{\infty} \hat{u}_{\infty} \mathrm{d} \hat{z}=\left(\frac{\hat{z}_{L}^{\infty}-\hat{b}_{\infty}}{\hat{h}_{\infty}}\right)^{2}>\frac{1}{(1+\lambda)^{2}}
$$

for all the incoming large particles to be deposited. In particular, if there is no deposition, $\lambda=0$, then there are no flow compositions that support a travelling-wave solution with the same speed as the front.

Assuming that the inequality (3.16) is satisfied it is possible to construct solutions in which the $\hat{\psi}_{L}$ particle path intersects the basal deposition surface. The complete structure of the solution is illustrated schematically in figure 7 and has many similarities with the lens-like solution shown in figure 4. There is an expansion fan centred at point $\mathrm{A}$, which lies on the no-mean-flow line $\hat{\psi}=\hat{\psi}_{u_{F}}$. This expands into the upper domain of material moving towards the flow front, and the concentration within the fan is

$$
\phi=\frac{1}{2}\left(1+\frac{\hat{\psi}-\hat{\psi}_{A}}{S_{r}\left(\hat{\xi}-\hat{\xi}_{A}\right)}\right),
$$

where $\left(\hat{\xi}_{A}, \hat{\psi}_{A}\right)$ is the position of point $\mathrm{A}$ in streamfunction coordinates. The leading $\phi=1$ characteristic $\mathrm{AB}$ is given by the line

$$
\hat{\psi}=\hat{\psi}_{A}+S_{r}\left(\hat{\xi}-\hat{\xi}_{A}\right),
$$

and this intersects the inversely graded layer $\hat{\psi}=\hat{\psi}_{L}$ at point B

$$
\hat{\xi}_{B}=\hat{\xi}_{A}+\frac{\hat{\psi}_{L}-\hat{\psi}_{A}}{S_{r}} .
$$




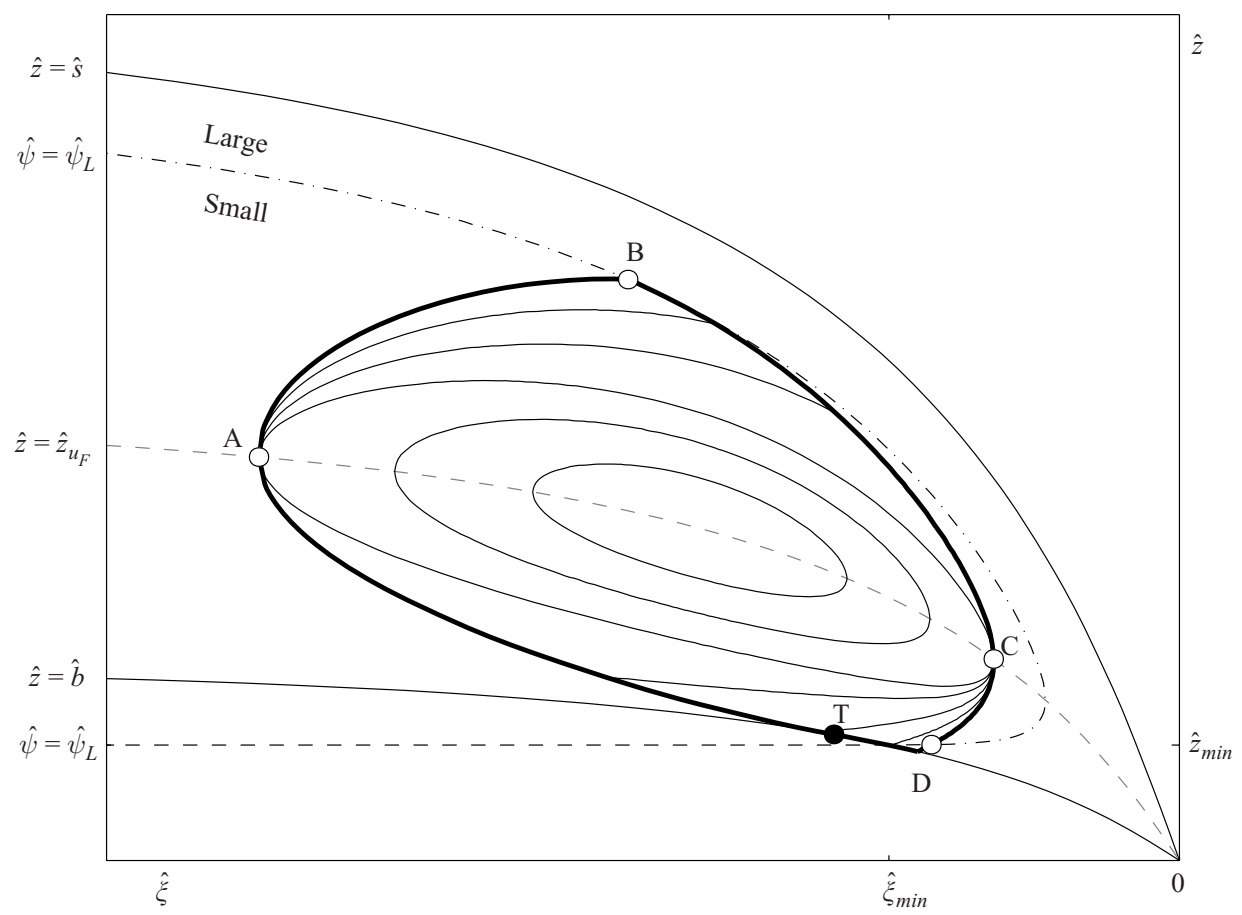

FIGURE 7. A diagram showing the position of the breaking size-segregation wave relative to the flow front. The large particles are separated from the small grains below, by a shock that lies along the $\hat{\psi}=\hat{\psi}_{L}$ particle path in the upper domain. The upper expansion fan is centred at point A (thin solid lines) above the no-mean-flow line $\hat{z}=\hat{z}_{u_{F}}$ (grey dashed line). The $\phi=1$ characteristic $\mathrm{AB}$ intersects the $\hat{\psi}_{L}$ particle path at point $\mathrm{B}$, and a shock $\mathrm{BC}$ is generated. As the shock reaches the no-mean-flow line at point $\mathrm{C}$ it forms a lower expansion fan (thin solid lines) below $\hat{z}_{u_{F}}$. The $\phi=0$ characteristic CD intersects with the $\psi_{L}$ particle path in the lower domain at point $\mathrm{D}$, and the large-particle path generated from $\mathrm{D}$ meets up again with the centred expansion at point A. At the centre of the breaking wave is an eye of constant concentration, in which the characteristics form closed lozenge-shaped curves (thin lines). There is a unique position for the breaking size-segregation wave, which requires that the large-particle path DA just touches the basal deposition interface $\hat{z}=\hat{b}$ at a tangent point $\mathrm{T}$ (black circle). The large-particle path TA is also a shock. A good approximation for the positions of both $\mathrm{D}$ and $\mathrm{T}$ is the point $\left(\hat{\xi}_{\text {min }}, \hat{z}_{\text {min }}\right)$, where the particle path $\psi_{L}$ intersects with $\hat{b}$.

Since there are no more small particles above $\hat{\psi}_{L}$ to percolate downwards, a shock $\mathrm{BC}$ is generated between the expansion fan (3.18) on one side and a pure phase of large particles on the other. The shock condition (3.11) yields the linear ordinary differential equation

$$
\frac{\mathrm{d} \hat{\psi}}{\mathrm{d} \hat{\xi}}=\frac{1}{2}\left(\frac{\hat{\psi}-\hat{\psi}_{A}}{\hat{\xi}-\hat{\xi}_{A}}-S_{r}\right) .
$$

This can be solved subject to condition that it starts from $\left(\hat{\xi}_{B}, \hat{\psi}_{L}\right)$ to give

$$
\hat{\psi}=\hat{\psi}_{A}-S_{r}\left(\hat{\xi}-\hat{\xi}_{A}\right)+2 \sqrt{\hat{\psi}_{L}-\hat{\psi}_{A}} \sqrt{S_{r}\left(\hat{\xi}-\hat{\xi}_{A}\right)} .
$$

The upper shock BC starts at $\left(\hat{\xi}_{B}, \hat{\psi}_{L}\right)$ and propagates downwards, reaching the nomean-flow line at $\left(\hat{\xi}_{C}, \hat{\psi}_{C}\right)$. Below $\hat{\psi}=\hat{\psi}_{u_{F}}$ the flow direction, and hence the time-like 
direction for the characteristics, reverses. Theorem 3.1 of Shearer et al. (2008) implies that the continuation of the shock in the lower domain does not satisfy the Lax entropy condition, and instead it breaks into a fan centred at $\left(\hat{\xi}_{C}, \hat{\psi}_{C}\right)$ in which the concentration

$$
\phi=\frac{1}{2}\left(1-\frac{\hat{\psi}-\hat{\psi}_{C}}{S_{r}\left(\hat{\xi}_{C}-\hat{\xi}\right)}\right) .
$$

The lead $\phi=0$ characteristic CD lies along the line

$$
\hat{\psi}=\hat{\psi}_{C}+S_{r}\left(\hat{\xi}_{C}-\hat{\xi}\right)
$$

and intersects the $\hat{\psi}=\hat{\psi}_{L}$ particle path again at point $\mathrm{D}$,

$$
\hat{\xi}_{D}=\hat{\xi}_{C}-\frac{\hat{\psi}_{L}-\hat{\psi}_{C}}{S_{r}}
$$

For steady states the breaking segregation wave recirculates large particles on the $\hat{\psi}_{L}$ particle path. Part of the path DA also forms a shock across which the small particles separate out, but since the equations for this shock and particle path are identical, it is not necessary to determine the location of the transition until later. The large-particle path equation (3.14) within the fan (3.23) becomes

$$
\frac{\mathrm{d} \hat{\psi}}{\mathrm{d} \hat{\xi}}=\frac{1}{2}\left(S_{r}-\frac{\hat{\psi}-\hat{\psi}_{C}}{\hat{\xi}_{C}-\hat{\xi}}\right)
$$

This can be solved subject to the condition that it starts at $\left(\hat{\xi}_{D}, \hat{\psi}_{L}\right)$ to show that

$$
\hat{\psi}=\hat{\psi}_{C}-S_{r}\left(\hat{\xi}_{C}-\hat{\xi}\right)+2 \sqrt{\hat{\psi}_{L}-\hat{\psi}_{C}} \sqrt{S_{r}\left(\hat{\xi}_{C}-\hat{\xi}\right)} .
$$

This intersects the no-mean-flow line $\hat{\psi}=\hat{\psi}_{u_{F}}$ again at $\left(\hat{\xi}_{A}, \hat{\psi}_{A}\right)$. The continuation of the shock into the upper domain is unstable by the Lax entropy condition (Shearer et al. 2008), and it forms an expansion fan centred at $\left(\hat{\xi}_{A}, \hat{\psi}_{A}\right)$, consistent with our original assumption in (3.18). The combination of the two expansion fans and two shock waves forms a structure that Thornton \& Gray (2008) termed a breaking size-segregation wave or 'lens'. There is an interesting new feature in this case. Since $\hat{\psi}_{A} \neq \hat{\psi}_{C}$, there is an additional central 'eye' of constant concentration,

$$
\phi_{\text {eye }}=\frac{1}{2}\left(1+\frac{\hat{\psi}_{C}-\hat{\psi}_{A}}{S_{r}\left(\hat{\xi}_{C}-\hat{\xi}_{A}\right)}\right),
$$

that is bounded above and below by the characteristics

$$
\begin{aligned}
& \hat{\psi}_{\text {eye }}^{\text {upper }}=\hat{\psi}_{A}+\left(2 \phi_{\text {eye }}-1\right) S_{r}\left(\hat{\xi}-\hat{\xi}_{A}\right), \\
& \hat{\psi}_{\text {eye }}^{\text {lower }}=\hat{\psi}_{C}-\left(2 \phi_{\text {eye }}-1\right) S_{r}\left(\hat{\xi}_{C}-\hat{\xi}\right) .
\end{aligned}
$$

By subtracting (3.29) from (3.30) and using (3.28), it is easy to show that $\hat{\psi}_{\text {eye }}^{\text {upper }}$ is equal to $\hat{\psi}_{\text {eye }}^{\text {lower }}$ for all values of $\hat{\xi}$. In streamfunction coordinates, the upper and lower boundaries of the central eye are therefore identical, except that one is located in the upper domain, and the other is in the lower one. Within the central eye the characteristics form closed lozenge-shaped curves as shown in figure 7.

A contour plot of the small-particle concentration is illustrated in figure 8. This shows that there are higher concentrations of large particles at the bottom left of the 


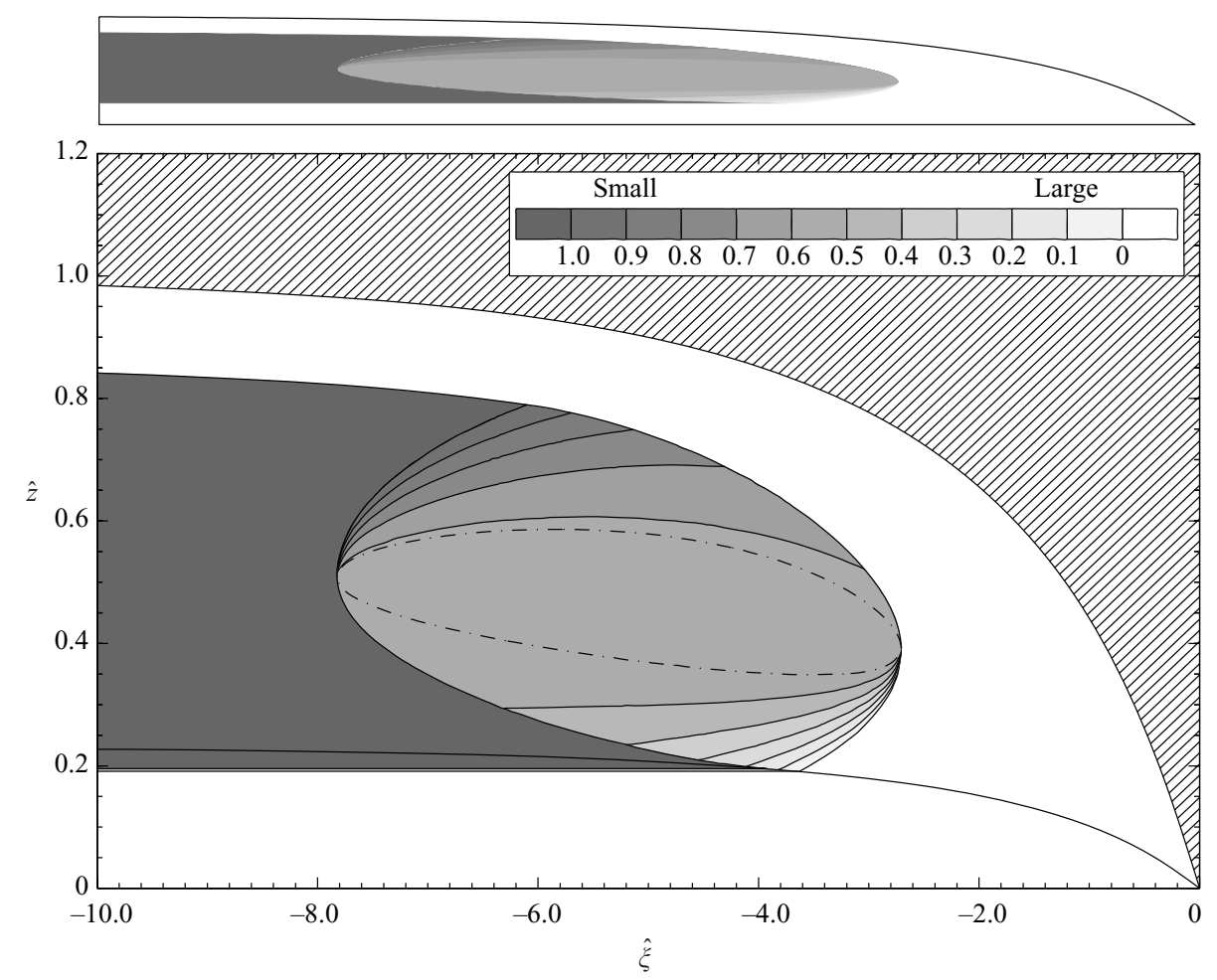

FIGURE 8. A contour plot of the small-particle concentration $\phi$ as a function of $(\hat{\xi}, \hat{z})$ for the bulk flow illustrated in figure 6 . White regions correspond to large particles and dark grey to pure fine, and there is a continuous scale in between (inset). The large and small particles enter from the top left and are separated by a shock along $\hat{\psi}_{L}=-0.193$. This value of is related to the composition of the mixture and corresponds to a height $\hat{z}_{L}^{\infty}=0.858$ as $\hat{\xi} \rightarrow-\infty$. The non-dimensional segregation number $S_{r}=0.1$. The central eye of the breaking wave is indicated by the dot-dashed line, and above the main figure is a compressed image showing the solution in a more realistic aspect ratio.

lens, and it becomes increasingly dominated by small particles towards the top right. Above the main part of figure 8 is a compressed version, which shows the solution in a more realistic aspect ratio. This has many features in common with the photo of the stratification experiment in figure $2(a)$. There is a flow front that is entirely composed of large particles, which propagates downslope at constant speed. Coarse grains that are sheared, by the bulk flow, towards the front pass over the top of the breaking size-segregation wave, are overrun and then deposited to form a stationary layer at the base. Upstream of the breaking wave, the avalanche concentration distribution resembles a small-particle sandwich, with a layer of rapidly flowing large particles at the top and a layer of deposited coarse grains at the bottom. Just as in the experiment, the upper and lower large-particle layers are almost the same thickness as they move towards and away from the moving front. The upper layer is slightly thinner, due to mass balance. Hyperbolic segregation theory is therefore able to capture the gross features of the particle-size distribution in the experimental avalanche. However, sharp shocks may be smoothed by diffusive remixing (Gray \& Chugunov 2006), and the discreteness of the physical system obscures the fine mathematical structure of the breaking wave in figure $2(a)$. There may also be other effects such as unsteadiness, 
out-of-plane motion, polydispersity and variations in the segregation rate $S_{r}$ and/or the solids volume fraction $v$ that complicate this still further. Nevertheless some form of breaking wave must exist, in order to join the coarse-particle front with the inversely graded flow behind and recirculate the large particles. Discrete numerical simulations may be able to give some further insights here, but, to date, existing computations have been done using bidisperse disks on relatively short smooth beds (e.g. Linares-Guerrero et al. 2007), and they have not been looking explicitly for these types of structures.

\subsection{Location of the breaking size-segregation wave}

It will now be shown that, in a two-dimensional depositing flow, there is a unique position for the breaking size-segregation wave described in $\S 3.2$. There are two constraints on the position of the wave. If it lies too far upstream, the bottom of the wave intersects the basal deposition surface, violating the assumption that $S_{r}$ is equal to a non-zero constant, while if it is too far downstream, not enough large particles are deposited, and some of them are drawn into the lens driving a time-dependent readjustment. For a steadily travelling wave to exist, the $\hat{\psi}_{L}$ large-particle path must just touch the deposition surface, $\hat{\psi}_{b}$; i.e. there is a unique tangency point $\left(\hat{\xi}_{T}, \hat{\psi}_{T}\right)$ on the particle path (3.27) at which

$$
\begin{aligned}
& \hat{\psi}\left(\hat{\xi}_{T}\right)=\hat{\psi}_{b}\left(\hat{\xi}_{T}\right), \\
& \left.\frac{\mathrm{d} \hat{\psi}}{\mathrm{d} \hat{\xi}}\right|_{\hat{\xi}_{T}}=\left.\frac{\mathrm{d} \hat{\psi}_{b}}{\mathrm{~d} \hat{\xi}}\right|_{\hat{\xi}_{T}},
\end{aligned}
$$

where $\hat{\psi}_{b}$ is the function of $\hat{s}$ given in (2.36). Although it is easy to state the conditions (3.31) and (3.32), there is no closed form solution, and instead one must iterate for its position. The simplest way to do this is to iterate for the free-surface height $\hat{s}_{C}$, where the lower expansion fan is located. This has the advantage that once $\hat{s}_{C}$ is known, the fan position $\left(\hat{\xi}_{C}, \hat{z}_{C}\right)$ can be recovered explicitly by (2.20) and (2.37). Figure 9 shows a close-up diagram of the base of the lens and the constructions necessary to find $\hat{s}_{C}$. A useful lower bound for $\hat{s}_{C}$ is the free-surface height at which the bulk-particle path $\hat{\psi}_{L}$ reaches its maximum downstream distance. This occurs when it intersects the no-mean-flow line $\hat{\psi}_{u_{F}}$, and the corresponding free-surface height and position are given by

$$
\hat{s}_{\max }=\frac{\hat{\psi}_{L}}{\lambda^{2}-\kappa^{2} / 4}, \quad \hat{\xi}_{\max }=\hat{\xi}\left(\hat{s}_{\max }\right), \quad \hat{z}_{\max }=\frac{\kappa}{2(1+\lambda)} \hat{s}_{\max } .
$$

A good first estimate for the position of the tangency point is where the $\hat{\psi}_{L}$ bulkparticle path intersects the basal deposition interface at

$$
\hat{s}_{\text {min }}=-\frac{1+\lambda}{\lambda} \hat{\psi}_{L}, \quad \hat{\xi}_{\text {min }}=\hat{\xi}\left(\hat{s}_{\text {min }}\right), \quad \hat{z}_{\text {min }}=\frac{\lambda}{1+\lambda} \hat{s}_{\text {min }}
$$

Projecting the $\phi=0$ characteristic curve (3.24), which passes through $\left(\hat{\xi}_{\text {min }}, \hat{\psi}_{L}\right)$, back onto the zero-mean-flow line gives the minimum position of $\left(\hat{\xi}_{C}, \hat{\psi}_{C}\right)$, which is termed $\left(\hat{\xi}_{C \min }, \hat{\psi}_{C \min }\right)$. This can be found by iterating for the free-surface height $\hat{s}_{C_{\text {min }}} \in\left[\hat{s}_{\text {min }}, \hat{s}_{\text {max }}\right]$ with

$$
\hat{\psi}_{L}-S_{r}\left(\hat{\xi}\left(\hat{s}_{C_{m i n}}\right)-\hat{\xi}_{m i n}\right)=\left(\lambda^{2}-\kappa^{2} / 4\right) \hat{s}_{C_{m i n}} .
$$




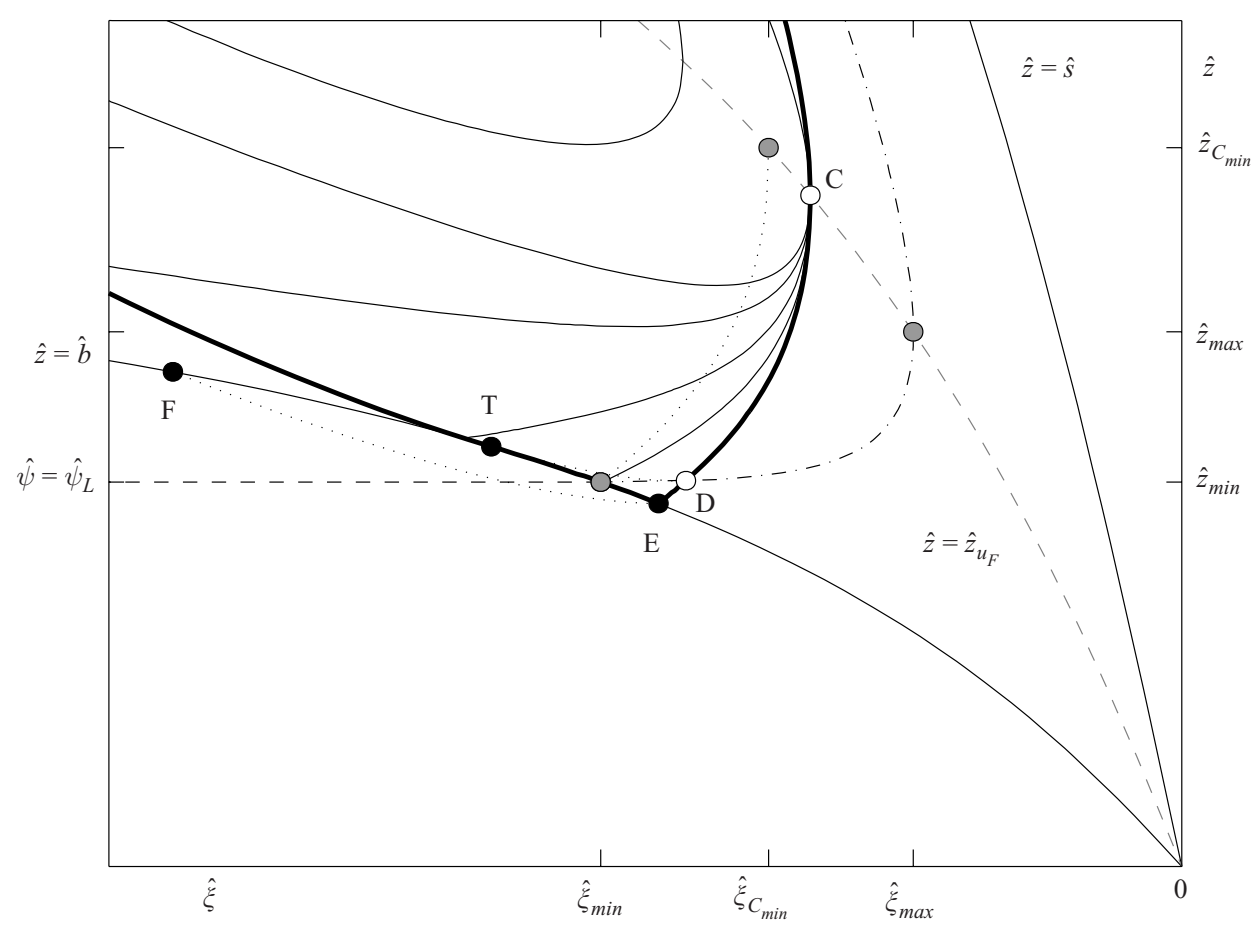

FIGURE 9. A close-up diagram of the intersection between the breaking size-segregation wave (thick solid line) and the basal deposition surface $\hat{z}=\hat{b}$. The lower expansion fan is centred at point $\mathrm{C}$ and lies on the (grey dashed) no-mean-flow line $\hat{z}=\hat{z}_{u_{F}}$. The lower part of the wave is bounded by the $\phi=0$ characteristic curve CE. This intersects the $\hat{\psi}_{L}$ particle path (dash-dotted line) at point D and the basal surface at point E. An iteration must be performed to find the $\hat{\xi}$-coordinate of the fan, $\hat{\xi}_{C}$. Since point E cannot be less than $\hat{\xi}_{\text {min }}, \hat{\xi}_{C}$ cannot be less than $\hat{\xi}_{C \text { min }}$, where $\hat{\xi}_{\text {Cmin }}$ is the projection (dotted line) of the $\phi=0$ characteristic curve that passes through $\hat{\xi}_{\text {min }}$ back up to the no-mean-flow line. An upper bound for $\hat{\xi}_{C}$ is determined by the maximum downstream distance $\hat{\xi}_{\text {max }}$ of the $\hat{\psi}_{L}$ particle path. Point $\mathrm{C}$ must be located so that the large-particle path emanating from point $\mathrm{D}$ just touches the basal deposition surface $\hat{z}=\hat{b}$ at point $\mathrm{T}$. The tangent point $\mathrm{T}$ lies between points $\mathrm{E}$ and $\mathrm{F}$, where $\mathrm{F}$ is the projection of a shock starting at E.

The free-surface height $\hat{s}_{C}$ therefore lies in the range $\left[s_{\max }, s_{C_{\min }}\right]$, and the iteration proceeds as follows: First construct point $\mathrm{E}$ at which the $\phi=0$ characteristic intersects the basal topography by iterating for $\hat{s}_{E} \in\left[s_{\min }, s_{\max }\right]$ using

$$
\hat{\psi}_{C}+S_{r}\left(\hat{\xi}_{C}-\hat{\xi}\left(\hat{s}_{E}\right)\right)=-\frac{\lambda}{1+\lambda} \hat{s}_{E}=\hat{\psi}_{E}
$$

This is an upper bound for the tangent. A lower bound is given by point F, where the large-particle path starting at $\left(\hat{\xi}_{E}, \hat{\psi}_{E}\right)$ would have intersected the basal topography, if $S_{r} \neq 0$ in the deposition region. This can be found by iterating for $\hat{s}_{F} \in\left(s_{E}, 1\right]$ with

$$
\hat{\psi}_{C}-S_{r}\left(\hat{\xi}_{C}-\hat{\xi}\left(\hat{s}_{F}\right)\right)+2 \sqrt{\hat{\psi}_{E}-\hat{\psi}_{C}} \sqrt{S_{r}\left(\hat{\xi}_{C}-\hat{\xi}\left(\hat{s}_{F}\right)\right)}=-\frac{\lambda}{1+\lambda} \hat{s}_{F}=\hat{\psi}_{F} .
$$

The tangent $\hat{s}_{T}$ therefore lies in the range $\left[\hat{s}_{E}, \hat{s}_{F}\right]$. Substituting (2.36), (3.26) and (3.31) into the tangency condition (3.32) yields an equation that can be used to iterate 


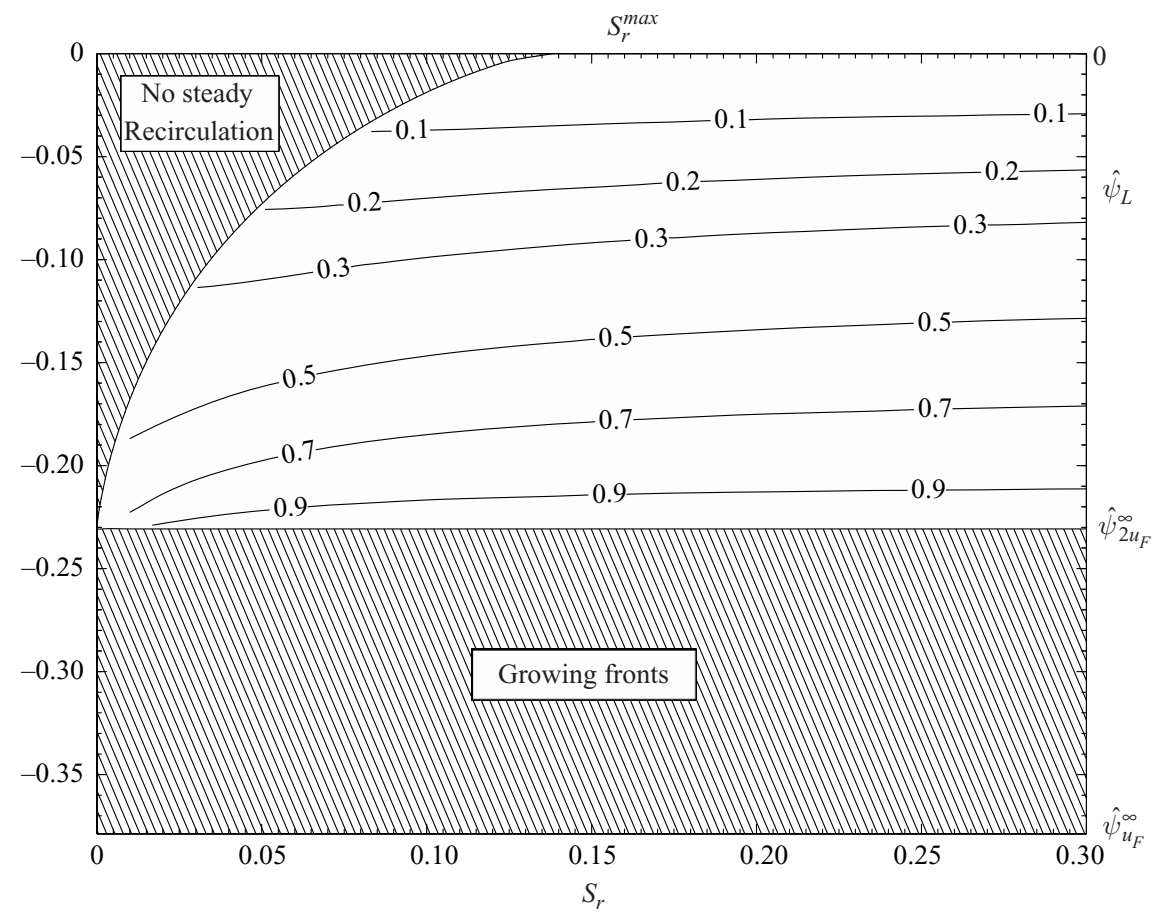

Figure 10. A contour plot of the equivalent free-surface height $\hat{s}_{C}$ at which the lower expansion fan is located, as a function of the non-dimensional segregation number $S_{r}$ and the particle path of the incoming shock $\psi_{L}$. There are no steadily recirculating and depositing solutions for paths $\psi_{L}$ below $\hat{\psi}_{2 u_{F}}^{\infty}$. There is also a spandrel-shaped region in the top left-hand corner, where the segregation is not strong enough for the particles to recirculate, and they are steadily deposited instead. The curves are constructed for a bulk flow profile $\gamma=1.5$ and deposition strength $\lambda=0.3$.

for $\hat{s}_{T}$,

$$
S_{r}+\frac{\hat{\psi}_{C}-\psi_{b}\left(\hat{s}_{T}\right)}{\hat{\xi}_{C}-\hat{\xi}\left(\hat{s}_{T}\right)}+\left.\frac{2 \lambda}{1+\lambda} \frac{\mathrm{d} \hat{s}}{\mathrm{~d} \hat{\xi}}\right|_{\hat{s}_{T}}=0,
$$

using the exact expression (2.19) for the free-surface gradient. The overall iteration for $\hat{s}_{C}$ converges when the large-particle path (3.27) and the basal topography (2.36) are equal at the tangency point $\hat{s}_{T}$; that is when the intersection condition (3.31) is satisfied

$$
\hat{\psi}_{C}-S_{r}\left(\hat{\xi}_{C}-\hat{\xi}_{T}\right)+2 \sqrt{\hat{\psi}_{L}-\hat{\psi}_{C}} \sqrt{S_{r}\left(\hat{\xi}_{C}-\hat{\xi}_{T}\right)}=-\frac{\lambda}{1+\lambda} \hat{s}_{T}=\hat{\psi}_{T},
$$

where $\hat{\xi}_{T}=\hat{\xi}\left(\hat{s}_{T}\right)$. The algorithm described above is complicated but guarantees convergence for all valid parameter values. Figure 10 shows the equivalent free-surface height $\hat{s}_{C}$ of the lower expansion fan, as a function of the non-dimensional segregation number $S_{r}$ and the incoming inversely graded shock path $\hat{\psi}_{L}$ in streamfunction coordinates. The location of the lower expansion is relatively insensitive to the value of $S_{r}$ but varies strongly with $\hat{\psi}_{L}$. If there are relatively few large particles, $\hat{\psi}_{L}$ is close to zero; $\hat{s}_{C}$ is small; and the breaking size-segregation wave is close to the flow front. As the number of larger particles increases, $\psi_{L}$ becomes increasingly negative, 


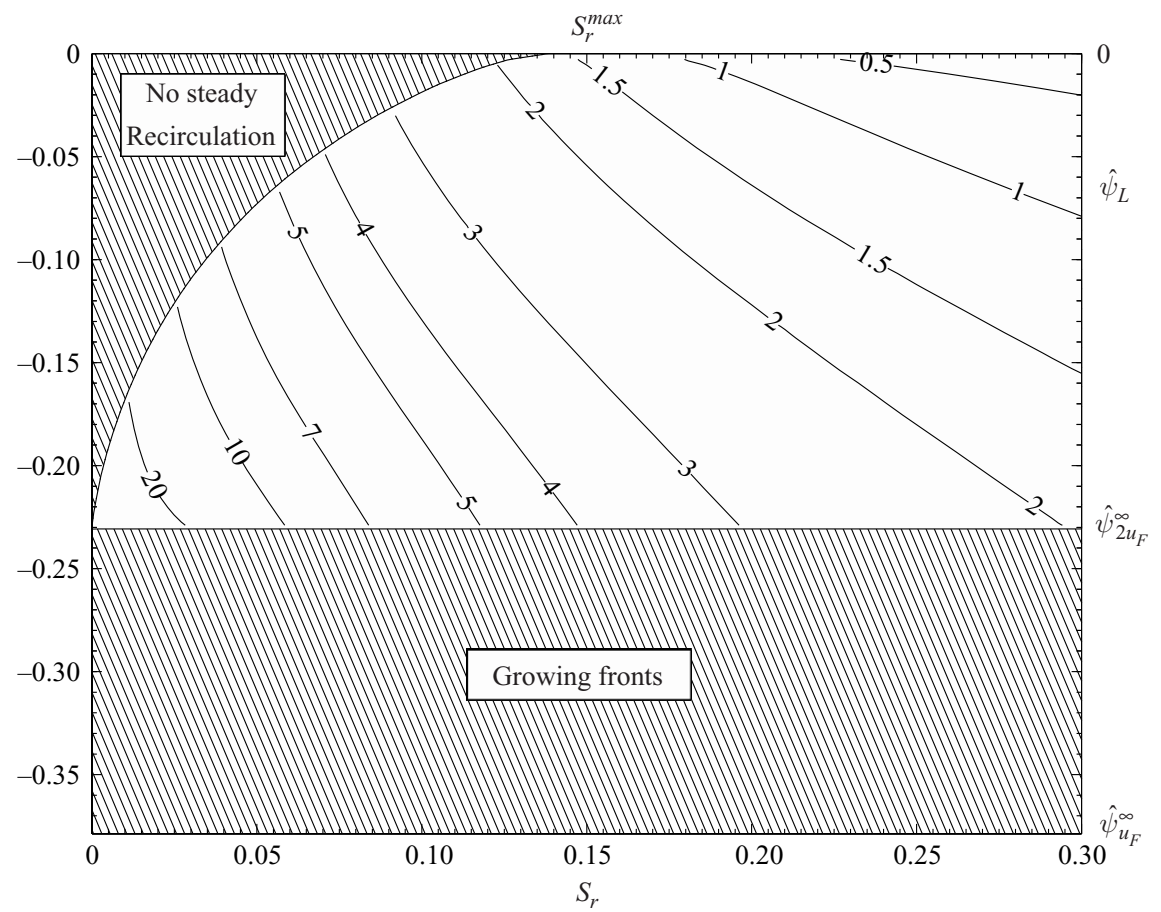

FIGURE 11. A contour plot of the breaking size-segregation wave width $\hat{\xi}_{C}-\hat{\xi}_{A}$, as a function of the non-dimensional segregation number $S_{r}$ and the particle path of the incoming shock $\psi_{L}$. All other quantities are the same as figure 10.

and the free-surface height $\hat{s}_{C}$ rises towards unity, implying that the lens is located further upstream. In the limit $\hat{\psi}_{L} \rightarrow \hat{\psi}_{2 u_{F}}^{\infty}, \hat{s}_{C} \rightarrow 1$, and the lens position $\hat{\xi}_{C}$ tends to minus infinity. For $\hat{\psi}_{L}<\hat{\psi}_{2 u_{F}}^{\infty}$ there are no steady solutions, and the size of the large-particle-rich front grows indefinitely. The existence and location of the breaking size-segregation wave are therefore strongly linked to the composition of the flow and the amount of deposition taking place.

Once $\hat{s}_{C}$ has been determined, it is relatively easy to iterate for the equivalent free-surface height $\hat{s}_{A} \in\left(\hat{s}_{C}, 1\right]$ of the upper fan. Evaluating (3.22) at $\hat{\xi}_{C}$ and (3.27) at $\hat{\xi}_{A}$ and summing yields an expression for the lens width that can be used in the iteration

$$
\hat{\xi}_{C}-\hat{\xi}_{A}=\frac{1}{S_{r}}\left(\sqrt{\hat{\psi}_{L}-\hat{\psi}_{C}}+\sqrt{\hat{\psi}_{L}-\hat{\psi}_{A}}\right)^{2} .
$$

A contour plot showing how the lens width varies with $S_{r}$ and $\hat{\psi}_{L}$ is shown in figure 11. Within the region in which solutions exist, the lens width decreases with increasing segregation number $S_{r}$ and with decreasing numbers of incoming large particles.

\subsection{Steadily depositing solutions}

In figures 10 and 11 there is a spandrel-shaped region, in the top left corner, where there are no steady recirculating solutions. This is because when $\hat{\psi}_{C}=\hat{\psi}_{L},(3.28)$ and (3.40) imply that the concentration in the central eye

$$
\phi_{\text {eye }}=1 \text {, }
$$




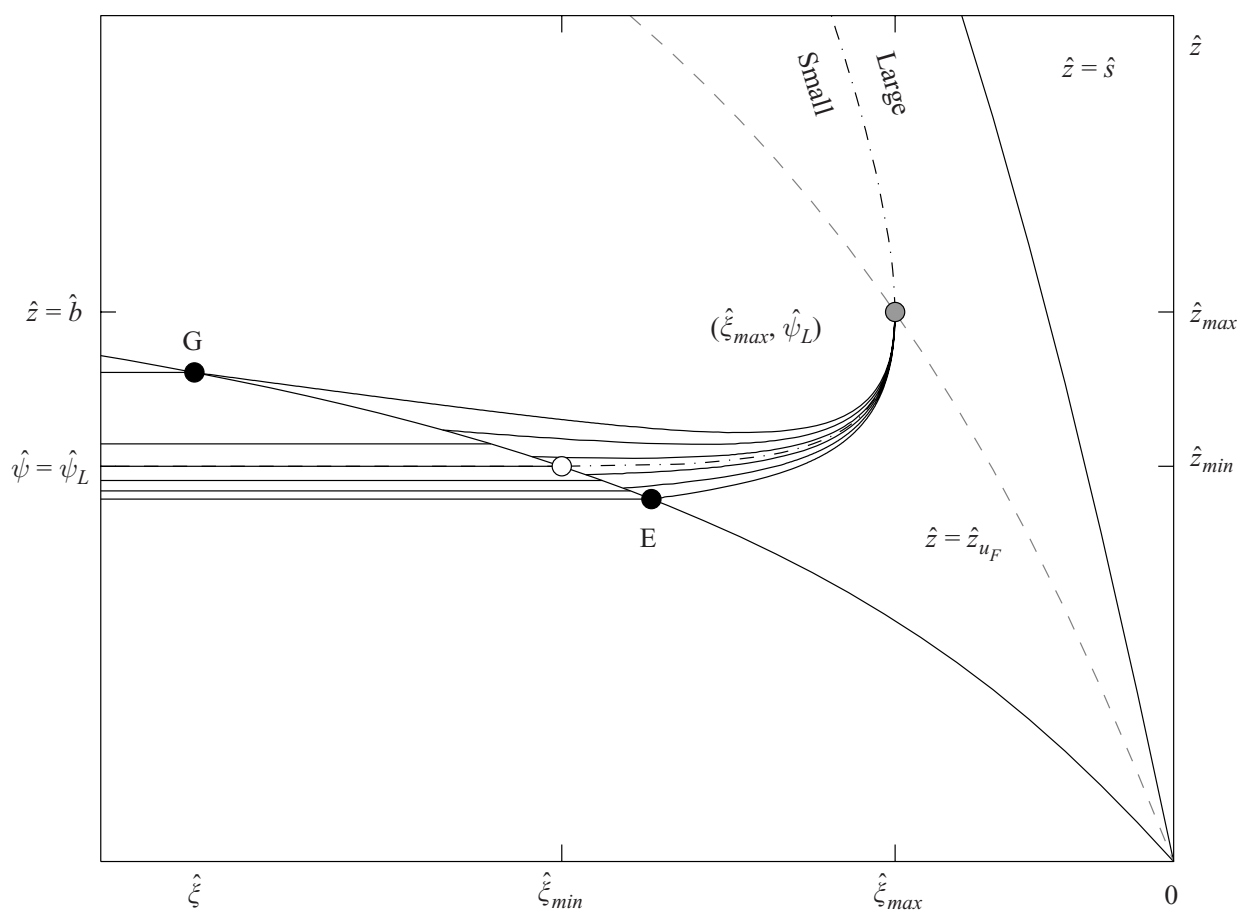

FIGURE 12. A close-up diagram showing that in the limit of low segregation number $S_{r}$ there is a second type of solution, in which all the large particles are deposited, and there is no recirculation. The large particles are separated from the small grains by a shock that lies along the $\hat{\psi}_{L}$ particle path in the upper domain. When this crosses the no-mean-flow line $\hat{z}_{u_{F}}$ it breaks to form an expansion fan centred at $\left(\hat{\xi}_{\max }, \hat{\psi}_{L}\right)$, and all the characteristics $\phi \in[0,1]$ emanating from it intersect the basal interface between points $\mathrm{E}$ and $\mathrm{G}$ (thin solid lines). There is a discontinuous jump in $S_{r}$ at the deposition surface $\hat{b}$, which implies that the deposition concentration $\phi^{+}$is greater than the incoming concentration $\phi^{-}$for $\phi^{-} \in(0,1)$. In the narrow deposition region between $\phi^{+}=0$ and $\phi^{+}=1$ there is therefore a discontinuity in $\phi$ at the basal interface, and the contours on either side do not match up.

and the structure of the breaking size-segregation wave degenerates. Physically, the segregation is not strong enough to cause the large particles to recirculate, and they are all deposited instead. Figure 12 shows a schematic diagram of the solution in this case. The incoming particles are assumed to be inversely graded with all the large ones above the $\hat{\psi}_{L}$ particle path. This propagates stably in the top domain but breaks as it crosses the no-mean-flow line $\hat{\psi}_{u_{F}}$, and there is a single centred expansion fan at $\left(\hat{\xi}_{\max }, \hat{\psi}_{L}\right)$ in which the concentration

$$
\phi=\frac{1}{2}\left(1-\frac{\hat{\psi}-\hat{\psi}_{L}}{S_{r}\left(\hat{\xi}_{\max }-\hat{\xi}\right)}\right)
$$

ranges from zero to unity. Provided the segregation is sufficiently weak, all the characteristics initiated at the expansion fan intersect the basal deposition surface $\hat{b}$, and the incoming large particles are deposited without recirculation.

The transition between the steadily recirculating and depositing solutions and the solutions that just steadily deposit, occurs when the $\phi=1$ characteristic emanating from the fan at $\left(\hat{\xi}_{\max }, \hat{\psi}_{L}\right)$ just touches the deposition surface; that is when the 
characteristic

$$
\hat{\psi}_{\text {char }}=\hat{\psi}_{L}-S_{r}\left(\hat{\xi}_{\text {max }}-\hat{\xi}\right)
$$

is equal and tangent to the basal surface

$$
\begin{aligned}
& \hat{\psi}_{\text {char }}\left(\hat{s}_{\mathscr{T}}\right)=\hat{\psi}_{b}\left(\hat{s}_{\mathscr{T}}\right), \\
& \left.\frac{\mathrm{d} \hat{\psi}_{\text {char }}}{\mathrm{d} \hat{\xi}}\right|_{\hat{s}_{\mathscr{T}}}=\left.\frac{\mathrm{d} \hat{\psi}_{b}}{\mathrm{~d} \hat{\xi}}\right|_{\hat{S}_{\mathscr{T}}},
\end{aligned}
$$

at the equivalent free-surface height $\hat{s}_{\mathscr{T}}$ of the tangency point. Substituting (2.19), (2.36) and (3.43) into the tangency condition (3.45) and solving for $\hat{s}_{\mathscr{T}}$ implies that

$$
s_{\mathscr{T}}=\left[\frac{1}{\gamma}\left(\frac{\lambda(1+\gamma)}{\lambda+(1+\lambda)(1+\gamma) S_{r}}-1\right)\right]^{\frac{2}{3}} .
$$

This is real, provided that the segregation number $S_{r}$ is less than or equal to

$$
S_{r}^{\max }=\frac{\gamma \lambda}{(1+\gamma)(1+\lambda)} .
$$

For a given inversely graded shock height $\hat{\psi}_{L}$, the critical value of the segregation number at which the solution switches from steadily recirculating and depositing to steadily depositing is found by iterating for $S_{r}^{c r i t} \in\left[0, S_{r}^{\max }\right]$ using condition (3.44), i.e.

$$
\hat{\psi}_{L}-S_{r}^{c r i t}\left(\hat{\xi}_{\max }-\hat{\xi}_{\mathscr{T}}\right)=-\frac{\lambda}{1+\lambda} \hat{S}_{\mathscr{T}}
$$

where $\hat{\xi}_{\mathscr{T}}=\xi\left(\hat{S}_{\mathscr{T}}\right)$. The curve $S_{r}=S_{r}^{\text {crit }}\left(\psi_{L}\right)$ determines the boundary of the spandrel shaped region in the upper left-hand corner of figures 10 and 11. If $S_{r}>S_{r}^{c r i t}$, then the solution is the steadily propagating breaking size-segregation wave illustrated in figures 7 and 9 , while if $S_{r} \leqslant S_{r}^{\text {crit }}$ there is steady deposition as shown in figure 12 .

\subsection{Particle concentration in the deposition region}

At the basal deposition surface, the velocity is continuous, but there is a discontinuity in $S_{r}$, since $S_{r}=0$ below $\hat{z}=\hat{b}$. The segregation jump condition (3.9) with $\hat{u}_{F}=1$ implies that the concentration on the deposition ' + ' side of $\hat{b}$ is equal to

$$
\phi^{+}=\phi^{-}+C\left(\phi^{-}\left(1-\phi^{-}\right)\right) \text {, }
$$

where $\phi^{-}$is the incoming concentration and

$$
C=-S_{r} /(\partial \hat{b} / \partial \hat{\xi}) \geqslant 0
$$

The deposition concentration $\phi^{+}$is therefore greater than or equal to the incoming concentration $\phi^{-}$. Physically this is because on the rearward side of the discontinuity there is a flux of material due to transport and segregation, while on the forward side there is only transport. From (3.49) it is clear that when $\phi^{-}=0, \phi^{+}=0$ and when $\phi^{-}=1, \phi^{+}=1$. However, for the recirculating and depositing solutions there is a short section between points $\mathrm{E}$ and $\mathrm{T}$ in figure 9, where the fan (3.23) intersects the basal topography, and it is not immediately clear that $\phi^{+}$necessarily lies in the range $[0,1]$. Using the large-particle path equation (3.26) the concentration $\phi^{-}$can be expressed in terms of the streamfunction gradient, i.e.

$$
\phi^{-}=\frac{1}{2}\left(1-\frac{\hat{\psi}-\hat{\psi}_{C}}{S_{r}\left(\hat{\xi}_{C}-\hat{\xi}\right)}\right)=\frac{1}{S_{r}} \frac{\partial \hat{\psi}}{\partial \hat{\xi}} .
$$


It follows from the tangency condition (3.32) that at point $\mathrm{T}$ the concentration

$$
\phi_{T}^{-}=\left.\frac{1}{S_{r}} \frac{\partial \hat{\psi}_{b}}{\partial \hat{\xi}}\right|_{\hat{s}_{T}}=-\left.\frac{1}{S_{r}} \frac{\partial \hat{b}}{\partial \hat{\xi}}\right|_{\hat{s}_{T}}=\frac{1}{C} .
$$

For the recirculating and depositing solutions $C>1$. The incoming concentration $\phi^{-}$ between points $\mathrm{T}$ and $\mathrm{E}$ therefore decreases from $1 / C$ to zero. By definition $1-\phi^{-} \geqslant 0$ and by the above argument $1-C \phi^{-} \geqslant 0$, so the product is also greater than zero:

$$
\left(1-\phi^{-}\right)\left(1-C \phi^{-}\right) \geqslant 0 .
$$

Expanding out the terms and rearranging implies that the deposition concentration $\phi^{+}$is always less than or equal to unity

$$
\phi^{+}=\phi^{-}+C \phi^{-}\left(1-\phi^{-}\right) \leqslant 1 .
$$

Moreover, substituting the incoming concentration (3.50) at the tangency point $\mathrm{T}$ into the jump condition (3.47) implies that the deposition concentration is precisely unity,

$$
\phi_{T}^{+}=1,
$$

and at point $\mathrm{E}$ the deposition concentration is zero. There is therefore a narrow region between points $\mathrm{E}$ and $\mathrm{T}$, where the deposition concentration monotonically increases from zero to unity. In the steady solution illustrated in figure 8 the narrow region lies between the layer of pure coarse deposited grains and pure fine ones, upstream of the breaking wave, but it is so thin that it is barely visible and could easily be misinterpreted as a shock. The case $C=1$ corresponds to the critical situation, where the $\phi=1$ characteristic is tangent to the basal deposition surface $\hat{b}$, and for $C<1$ the solution degenerates to the purely depositing front discussed in $\S 3.4$ and shown in figure 12. In this situation the incoming concentration $\phi^{-} \in[0,1]$ and the inequalities (3.51) and (3.52) are still valid. There is therefore also a region, between points $\mathrm{E}$ and $\mathrm{G}$ in figure 12 , where the deposition concentration varies continuously between zero and unity, and this is indicated by a break in the concentration contours as they cross the basal deposition surface.

\subsection{Large- and small-particle recirculation}

Large grains have been observed to recirculate at coarse-particle-rich flow fronts (Pouliquen et al. 1997; Félix \& Thomas 2004), and this plays a key part in the development of frontal instabilities (Pouliquen \& Vallance 1999) and levee formation in geophysical mass flows (Iverson \& Vallance 2001; Iverson 2003). While several authors (Pouliquen et al. 1997; Pouliquen \& Vallance 1999; Vallance 2000; Vallance \& Savage 2000; Félix \& Thomas 2004) have drawn schematic diagrams of the recirculation, the exact solution developed here allows us to explicitly compute the structure of these recirculating loops. They can be parameterized by their path

$$
\hat{\psi}_{o} \in\left[\hat{\psi}_{C}, \hat{\psi}_{L}\right]
$$

through the coarse-grained front, and cross the ' 0 ' characteristic (3.24) between points $\mathrm{C}$ and $\mathrm{D}$ in figures 7 and 9 , at

$$
\hat{\xi}_{i n}=\hat{\xi}_{C}-\left(\hat{\psi}_{o}-\hat{\psi}_{C}\right) / S_{r} .
$$

The trajectory through the lower expansion (3.23) can be calculated by solving the linear ordinary differential equation (3.14), subject to the initial condition (3.57), to 
give

$$
\hat{\psi}=\hat{\psi}_{C}-S_{r}\left(\hat{\xi}_{C}-\hat{\xi}\right)+2 \sqrt{\hat{\psi}_{o}-\hat{\psi}_{C}} \sqrt{S_{r}\left(\hat{\xi}_{C}-\hat{\xi}\right)} .
$$

This is similar in form to the bottom shock (3.27) between points $\mathrm{T}$ and A in figure 7 and is precisely the same when $\hat{\psi}_{o}=\hat{\psi}_{L}$. The particle path (3.58) intersects the bottom of the central eye at point $\mathrm{P}$, which has coordinates

$$
\hat{\xi}_{P}=\hat{\xi}_{C}-\frac{\hat{\psi}_{o}-\hat{\psi}_{C}}{S_{r}\left(1-\phi_{\text {eye }}\right)^{2}}, \quad \hat{\psi}_{P}=\hat{\psi}_{C}-\frac{\left(2 \phi_{\text {eye }}-1\right)\left(\hat{\psi}_{o}-\hat{\psi}_{C}\right)}{\left(1-\phi_{\text {eye }}\right)^{2}} .
$$

Once in the eye, the concentration $\phi_{\text {eye }}$ given in (3.28) is constant. The trajectory through the lower part of the eye is

$$
\hat{\psi}=\hat{\psi}_{P}+\phi_{\text {eye }} S_{r}\left(\hat{\xi}-\hat{\xi}_{P}\right) .
$$

The eye's symmetry property ensures that exactly the same equation governs the path through the upper eye, above $\hat{\psi}_{u_{F}}$. It follows that point $\mathrm{Q}$, where the large particle crosses the upper boundary of the eye (3.29), has exactly the same streamfunction coordinates as the entry point, i.e.

$$
\hat{\xi}_{Q}=\hat{\xi}_{P}, \quad \hat{\psi}_{Q}=\hat{\psi}_{P} .
$$

Substituting (3.18) into (3.14) yields a linear ordinary differential equation for the trajectory through the top expansion. Using (3.29), (3.59) and (3.61) the solution can be written as

$$
\hat{\psi}=\hat{\psi}_{A}+S_{r}\left(\hat{\xi}-\hat{\xi}_{A}\right)-2\left(1-\phi_{\text {eye }}\right) \sqrt{S_{r}\left(\hat{\xi}_{P}-\hat{\xi}_{A}\right)} \sqrt{S_{r}\left(\hat{\xi}-\hat{\xi}_{A}\right)} .
$$

A more convenient form of this equation can be obtained by noting that the lower shock (3.27) and the lower boundary of the eye (3.30) intersect at $\hat{\xi}_{A}$, which implies

$$
\left(1-\phi_{\text {eye }}\right)^{2} S_{r}\left(\hat{\xi}_{C}-\hat{\xi}_{A}\right)=\psi_{L}-\psi_{C} .
$$

Adding and subtracting $\hat{\xi}_{P}$ within the second bracketed term on the left-hand side of (3.63) and using (3.59) it follows that

$$
\left(1-\phi_{\text {eye }}\right)^{2} S_{r}\left(\hat{\xi}_{P}-\hat{\xi}_{A}\right)=\psi_{L}-\psi_{o},
$$

and therefore that the trajectory through the upper expansion (3.62) is

$$
\hat{\psi}=\hat{\psi}_{A}+S_{r}\left(\hat{\xi}-\hat{\xi}_{A}\right)-2 \sqrt{S_{r}\left(\hat{\psi}_{L}-\hat{\psi}_{o}\right)} \sqrt{S_{r}\left(\hat{\xi}-\hat{\xi}_{A}\right)} .
$$

From this it is easy to see that (3.65) reduces to the equation of the $\phi=1$ characteristic (3.19) emanating from $\left(\hat{\xi}_{A}, \hat{\psi}_{A}\right)$ when $\hat{\psi}_{o}=\hat{\psi}_{L}$. The trajectory intersects with the top shock (3.22) at

$$
\hat{\xi}_{\text {out }}=\hat{\xi}_{A}+\frac{1}{S_{r}}\left(\sqrt{\hat{\psi}_{L}-\hat{\psi}_{o}}+\sqrt{\hat{\psi}_{L}-\hat{\psi}_{A}}\right)^{2}, \quad \hat{\psi}_{\text {out }}=\hat{\psi}_{o},
$$

forming a closed loop with the $\hat{\psi}_{o}$ path in the large region. A series of recirculating large-particle loops for $\hat{\psi}_{o} \in\left[\hat{\psi}_{C}, \hat{\psi}_{L}\right]$ are illustrated in figure 13. Large particles recirculate around these paths in a clockwise fashion. They are swept into the bottom right-hand side of the lens by the bulk flow and rise up through it by particle-size segregation, crossing the no-mean-flow line and exiting across the top shock, before being swept down to the bottom again by the bulk flow. Large-particle paths that start above the incoming shock path $\hat{\psi}_{L}$ are all deposited. Most of these paths remain 


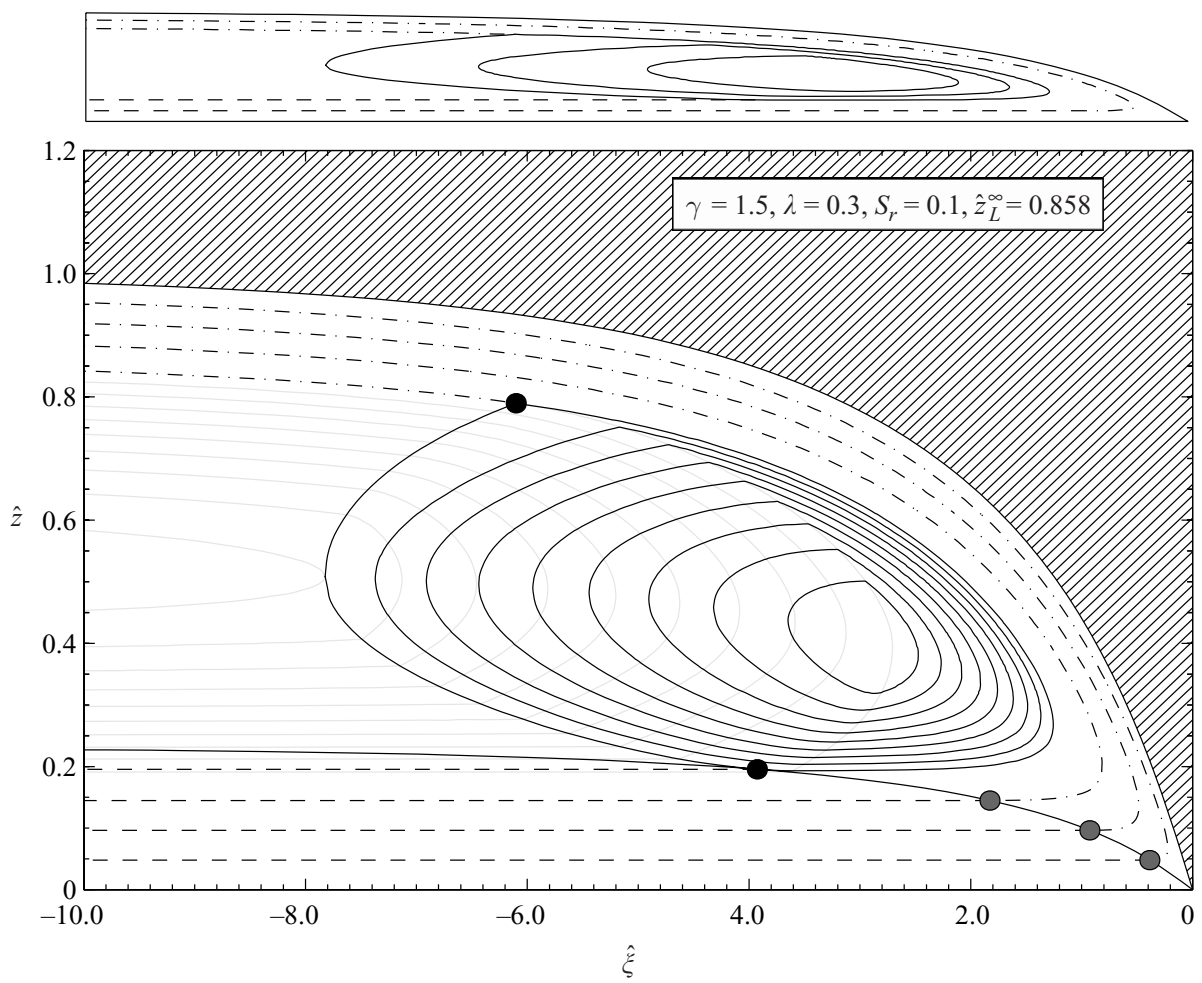

FIGURE 13. The large-particle paths are shown for the bulk flow and concentration solutions shown in figures 6 and 8. Particle paths that lie above $\hat{\psi}_{L}$ are all deposited as indicated by the dot-dashed and dashed lines and the grey circular markers. Most of these paths are the same as the bulk flow paths, as they remain in purely coarse-grained regions. However, there are a few that briefly enter the lower expansion fan before being deposited into the narrow region, where the concentration varies between zero and unity. The solid lines correspond to the paths of large grains that are recirculated at the flow front. They circulate clockwise, entering the breaking wave at the bottom right and rise up by particle-size segregation, before crossing the top shock and being swept around by the bulk flow to the bottom again. The $\hat{\psi}_{L}$ path marks the division between these two sets of paths, and the black markers indicate the top of the breaking wave $\hat{\xi}_{B}$ and the tangency point $\hat{\xi}_{T}$. Above the main figure is a compressed image showing the solution in a more realistic aspect ratio.

within the region of purely large particles, and they necessarily follow the bulk-particle paths (2.29). These paths are shown as dot-dashed and dashed lines separated by grey markers at the deposition point in figure 13. There are also a few large-particle paths that lie just above $\hat{\psi}_{L}$, which intersect the ' 0 ' characteristic $(3.24)$ between points D and $\mathrm{E}$ in figure 9. These grains follow the bulk-particle paths except for a very brief period when they move up through the expansion, before being deposited between points $\mathrm{E}$ and $\mathrm{T}$.

The fine ones are also recirculated in the clockwise sense, and their particle paths are shown in figure 13 using faint grey lines. Small grains enter from the top left and follow the bulk paths in the fine region as they are sheared towards the front. If the path $\hat{\psi}_{o}<\hat{\psi}_{A}$, then the particles recirculate before reaching the breaking wave. Paths in the range $\hat{\psi}_{o} \in\left[\psi_{A}, \psi_{C}\right]$ enter the breaking wave and are rapidly segregated downwards as large particles are driven upwards by squeeze expulsion. As they exit the breaking wave three things may happen: (i) they may cross the bottom shock

\section{CAmbridge JDURNALS}


and be recirculated backwards within the avalanche; (ii) they may exit across the shock and be transported a short distance, before being deposited; or (iii) they may deposit directly out of the breaking wave between points $\mathrm{E}$ and $\mathrm{T}$ in figure 9 . The explicit formulae are closely analogous to those obtained for the large particles in (3.56)-(3.66) and omitted for brevity.

\section{Discussion and conclusions}

The stratification experiments in $\S 1.1$ showed that large rough particles rise to the surface of the flow by particle-size segregation and are transported to the flow front by velocity shear. Here they experience much greater resistance to motion and are deposited on the underlying substrate, which allows the more mobile incoming material behind to progress further downslope. This is explicitly revealed in figure 2(a), which shows that there is a well-defined coarse-grained flow front, which is followed by a steady uniform flow, with a layer of small particles sandwiched between a rapidly moving layer of large particles at the surface and a static carpet of deposited coarse grains at the base. This complex structure has not been reported before, and it shows that there is a travelling-wave regime, where all the large rough particles that reach the front are deposited on the underlying slope. As a result the coarse-grained flow front stays at a constant length, instead of growing approximately linearly with time, as one would otherwise expect in the absence of deposition.

Stratification patterns are a prime example of flows in which the evolving particlesize distribution provides feedback to the mobility of the bulk flow and the deposition from it. This is termed the segregation-mobility feedback effect. Other examples include the formation of triangular segregation patterns in two-dimensional heaps (Herrmann 1998) and in three dimensions the formation of fingers and static lateral levees (Costa \& Williams 1984; Pierson 1986; Pouliquen et al. 1997; Pouliquen \& Vallance 1999; Iverson \& Vallance 2001; Jomelli \& Bertran 2001; Bertran 2003; Iverson 2003; Félix \& Thomas 2004; Iverson 2005; Aranson et al. 2006; Goujon et al. 2007).

A fully coupled solution is beyond the scope of this paper, but particle-size segregation models (e.g. Savage \& Lun 1988; Dolgunin \& Ukolov 1995; Gray \& Thornton 2005; Gray \& Chugunov 2006; Thornton et al. 2006) open up the realistic possibility of developing fully coupled theories in future. One way to achieve this is to use an avalanche model (e.g. Grigorian et al. 1967; Savage \& Hutter 1989; Iverson 1997; Gray et al. 1999; Pouliquen 1999a,b; Wieland et al. 1999; Denlinger \& Iverson 2001; Iverson \& Denlinger 2001; Gray et al. 2003) to (i) calculate the depthaveraged velocity $\overline{\boldsymbol{u}}$, (ii) use assumed velocity profiles through the avalanche depth to reconstruct the three-dimensional velocity field $\boldsymbol{u}$, (iii) compute the concentration $\phi$ with a segregation model and (iv) then couple them using a segregation-mobility feedback. There are several ways that this coupling may be achieved; the most straightforward is to $(a)$ modify the basal friction in response to the evolving flow composition (Pouliquen \& Vallance 1999), but one could also (b) change the assumed velocity profile through the avalanche (Phillips et al. 2006; Linares-Guerrero et al. 2007; Rognon et al. 2007) and/or (c) make the deposition rate composition dependent.

Given our relatively rudimentary knowledge of particle-size segregation, the primary aim of this paper has been to investigate the structure of the size distribution in a non-uniform depositing flow. A very simple uncoupled depth-averaged solution has been used to capture the basic kinematics of the avalanche motion observed in $\S 1.1$. The derivation illustrates the key stages at which full coupling could be achieved 
and where additional effects, such as sidewall friction, could be included. Exact solutions to the segregation problems in non-uniform depositing flows are due, in no small part, to the introduction of streamfunction coordinates in (2.32), which linearize the segregation equation (3.4), the jump condition (3.9) and the particle path equations (3.13). These coordinates are the natural extension of the depth-integrated velocity coordinates, which have been used extensively to solve segregation problems in steady uniform flows (Gray \& Thornton 2005; Gray et al. 2006; Thornton et al. 2006; McIntyre et al. 2008; Shearer et al. 2008; Thornton \& Gray 2008). Another significant generalization lies in the treatment of the concentration jump condition over the basal deposition interface in $\S 3.5$, where there is segregation on only one side. These types of interfaces are common in many problems, and this is likely to find application elsewhere.

Three types of segregation solution have been identified, which are dependent on the segregation rate $S_{r}$, the amount of deposition $\lambda$ and the incoming composition, which is set by the inversely graded shock path $\hat{\psi}_{L}$. The most interesting of these is when the segregation is sufficiently strong, and all the large particles that reach the front are deposited. In this situation a breaking size-segregation wave forms behind the front, which joins a downstream region of pure coarse grains to an upstream region in which the particle-size distribution resembles a small-particle sandwich. The small-particle concentration $\phi$ is shown in figure 8 and provides the first exact solution for the particle-size distribution in a non-uniform depositing flow. The breaking wave is more general than the uniform case (Thornton \& Gray 2008) and consists of two expansion fans, two shocks and a central eye of constant concentration, which are arranged in a lens-like structure that is located at a unique position behind the flow front. Hyperbolic segregation theory (Gray \& Thornton 2005; Thornton \& Gray 2008) therefore captures the gross features of the experimental flow shown in figure 2(a), such as the coarse-grained flow front, the raft of rapidly moving large particles at the surface, the static carpet of coarse grains deposited at the bottom and the small particles sandwiched in-between, but the finer structures in the breaking wave are hard to see and are lost in the graininess of these thin flows. Indeed it is even hard to identify these detailed features in the compressed image at the top of figure 8, which uses just a contour scale to represent the exact solution.

Another important feature of these solutions is that it is possible to explicitly track the large and small particles. Figure 13 shows how some of the large particles are recirculated at the flow front, while all of the coarse grains that are transported to the front are deposited. There are several conceptual diagrams of recirculation at flow fronts in the literature, and large recirculating particles have been observed in the field by Costa \& Williams (1984) and Pierson (1986) and measured in fingering experiments by Pouliquen et al. (1997) and Pouliquen \& Vallance (1999), but this paper provides the first concrete structure for it in a two-dimensional depositing flow. It should be noted that the solution is idealized in the sense that it is steady in the moving frame. Realistic flows are likely to have some element of unsteadiness, as well as other effects, such as diffusive remixing, polydispersity of the grain-size population, out-of-plane motion and discreteness, that break the idealized recirculation loops shown in figure 13 and allow large particles to have more complicated paths.

Finally, it should be noted that this travelling wave breaks down (i) either when the segregation rate is too low (ii) or when all the large incoming particles cannot be deposited. In the latter case, the large particles gradually accumulate at the flow front, and a specific example of this, in the absence of deposition, is shown in figure 4 . In fully coupled models these types of growing coarse-particle-rich fronts provide more 
and more resistance to motion and may ultimately bring the front to a complete halt. In contrast the unique location of the breaking wave in the travelling-wave solutions is rather surprising, and may be particularly significant for fully coupled models, as it determines the length scale over which there is enhanced basal friction.

This research was performed during three research visits to the Hydraulics Laboratory at EPFL, Lausanne, and was supported by generous funding from the School of Architecture, Civil and Environmental Engineering as well as the European Research Community on Flow, Turbulence and Combustion (ERCOFTAC). Nico Gray also acknowledges support from NERC grants NER/A/S/2003/00439 and NE/E003206/1, as well as an EPSRC Advanced Research Fellowship GR/S50052/01 and GR/S50069/01. Christophe Ancey is grateful for the financial support provided by the Swiss National Science Foundation under grant number 200021-105193, the competence centre in Mobile Information and Communication Systems (a centre supported by the Swiss National Science Foundation under grant number 5005-67322, MICS project) and the competence centre in Environmental Sciences (TRAMM project).

\section{REFERENCES}

Abramowitz, M. \& Stegun, I. 1970 Handbook of Mathematical Functions, 9th edn. Dover.

ANCEY, C. 2002 Dry granular flow down an inclined channel: experimental investigations on the frictional-collisional regime. Phys. Rev. E 65, 011304.

Aranson, I. S., Malloggi, F. \& Clement, E. 2006 Transverse instability in granular flows down an incline. Phys. Rev. E 73, 050302(R).

AzAnZA, E. 1998 Ecoulements granulaires bidimensionnels sur un plan incliné. Thèse l'École Nationale des Ponts et Chaussées, France, Spécialité: Structures et matériaux, 263 pp.

BAGNOLD, R. A. 1954 Experiments on gravity-free dispersion of large solid spheres in a Newtonian fluid under shear. Proc. R. Soc. Lond. A 225, 49-63.

Balmforth, N. J. \& Kerswell, R. R. 2005 Granular collapse in two dimensions. J. Fluid Mech. 538, 399-428.

Baran, O., Ertas, D. \& Halcey, T. C. 2006 Velocity correlations in dense gravity-driven granular chute flow. Phys. Rev. E 74, 051302, 1-10.

Bartelt, P., Buser, O. \& Platzer, K. 2007 Starving avalanches: frictional mechanisms at the tails of finite-sized mass movements. Geophys. Res. Lett. 34 (20), L20407.

Baxter, J., Tüzün, U., Heyes, D., Hayati, I. \& Fredlund, P. 1998 Stratification in poured granular heaps. Nature 391, 136.

Bertran, P. 2003 The rock-avalanche of February 1995 at Claix (French Alps). Geomorphology 54, 339-346.

Calder, E. S., Sparks, R. S. J. \& Gardeweg, M. C. 2000 Erosion, transport and segregation of pumice and lithic clasts in pyroclastic flows inferred from ignimbrite at Lascar Volcano, Chile. J. Volcan. Geotherm. Res. 104, 201-235.

Costa, J. E. \& Williams, G. P. 1984 Debris flow dynamics. US Geological Survey, Open-file Report, pp. 84-606. (22 minute video) http://pubs.er.usgs.gov/usgspubs/ofr/ofr84606.

Chadwick, P. 1999 Continuum mechanics: precise theory and problems (2nd corr. and enl. ed.), Dover $187 \mathrm{pp}$.

Cruden, D. M. \& Hungr, O. 1986 The debris of the Frank slide and theories of rockslide avalanche mobility. Can. J. Earth Sci. 33 (3).

Cui, X., Gray, J. M. N. T., Johannesson, T. 2007 Deflecting dams and the formation of oblique shocks in snow avalanches at Flateyri, Iceland. J. Geophys. Res. 112, F04012.

Daerr, A. 2001 Dynamical equilibrium of avalanches on a rough plane. Phys. Fluids 13, 2115-2124.

Deboeuf, S., Lajeunesse, E., Dauchot, O. \& Andreotti, B. 2006 Flow rule, self channelization, and levees in unconfined granular flows. Phys. Rev. Lett. 97, 158303. 
Denlinger, R. P. \& Iverson, R. M. 2001 Flow of variably fluidized granular masses across threedimensional terrain. Part 2. Numerical predictions and experimental tests. J. Geophys. Res. B1, 553-566.

Dolgunin, V. N. \& Ukolov, A. A. 1995 Segregation modelling of particle rapid gravity flow. Powder Technol. 83, 95-103.

Douady, S., Andreotti, B., Daerr, A. \& Cladé, E. 2002 From a grain to avalanches: on the physics of granular surface flows. C. R. Physique 3, 177-186.

Doyle, E. E., Huppert, H. E., Lube, G., Mader, H. M. \& Sparks, R. S. 2007 Static and flowing regions in granular collapses down channels: insights from a sedimenting shallow water model. Phys. Fluids 19, 106601.

Ehrichs, E. E., Jaeger, H. M., Karczmar, G. S., Knight, J. B., Kuperman, V. Y. \& Nagel, S. R. 1995 Granular convection observed by magnetic-resonance-imaging. Science 267 (5204), 16321634.

FéLIX, G. \& Thomas, N. 2004 Relation between dry granular flow regimes and morphology of deposits: formation of levées in pyroclastic deposits. Earth Planet. Sci. Lett. 221, 197-213.

GDR MiDi 2004 On dense granular flows. Eur. Phys. J. E 14, 341-365.

Goujon, C., Dalloz-Dubrujeaud, B. \& Thomas, N. 2007 Bidisperse granular avalanches on inclined planes: a rich variety of behaviours. Eur. Phys. J. E 23, 199-215.

GraY, J. M. N. T. 2001 Granular flow in partially filled slowly rotating drums. J. Fluid Mech. 441, $1-29$.

Gray, J. M. N. T. \& Chugunov, V. A. 2006 Particle-size segregation and diffusive remixing in shallow granular avalanches. J. Fluid Mech. 569, 365-398.

Gray, J. M. N. T. \& CUI, X. 2007 Weak, strong and detached oblique shocks in gravity driven granular free-surface flows. J. Fluid Mech. 579, 113-136.

Gray, J. M. N. T. \& HutTer, K. 1997 Pattern formation in granular avalanches. Continuum Mech. Thermodyn. 9, 341-345.

Gray, J. M. N. T. \& Hutter, K. 1998 Physik granularer Lawinen. Physikal. Blatter 54 (1), 37-43.

Gray, J. M. N. T., Shearer, M. \& Thornton, A. R. 2006 Time-dependent solutions for particle-size segregation in shallow granular avalanches. Proc. R. Soc. A 462, 947-972.

Gray, J. M. N. T. \& TAI, Y. C. 1998 Particle size segregation, granular shocks and stratification patterns. In Physics of Dry Granular Media (ed. H. J. Herrmann, J.-P. Hovi \& S. Luding), pp. 697-702, NATO ASI series. Kluwer Academic.

Gray, J. M. N. T., TaI, Y. C. \& NoElle, S. 2003 Shock waves, dead-zones and particle-free regions in rapid granular free-surface flows. J. Fluid Mech. 491, 161-181.

Gray, J. M. N. T. \& Thornton, A. R. 2005 A theory for particle size segregation in shallow granular free-surface flows. Proc. R. Soc. A 461, 1447-1473.

Gray, J. M. N. T., Wieland, M. \& Hutter, K. 1999 Free surface flow of cohesionless granular avalanches over complex basal topography. Proc. R. Soc. A 455, 1841-1874.

Grasselli, Y. \& Herrmann, H. J. 1997 On the angles of dry granular heaps. Physica A 246, 301-312.

Grigorian, S. S., Eglit, M. E. \& Iakimov, IU. L. 1967 New state and solution of the problem of the motion of snow avalance. Snow Avalanches Glaciers. Tr. Vysokogornogo Geofizich Inst. 12, 104-113.

Herrmann, H. J. 1998 On the shape of a sandpile. In Physics of dry granular media (ed. H. J. Herrmann, J.-P. Hovi \& S. Luding), pp. 697-702, NATO ASI series. Kluwer Academic.

Hill, K. M., Gioia, G. \& Amaravadi, D. 2004 Radial segregation patterns in rotating granular mixtures: waviness selection. Phys. Rev. Lett. 93, 224301, 1-4.

Hsiau, S. S. \& Hunt, M. L. 1993 Shear-induced particle diffusion and longitudinal velocity fluctuations in a granular-flow mixing layer. J. Fluid Mech. 251, 299-313.

ISSLER, D. 2003 Experimental information on the dynamics of dry snow avalanches. In Dynamic Response of Granular and Porous Material under Large and Catastrophic Deformations (ed. K. Hutter \& N. Kirchner), vol. 11, pp. 251-261, Lecture Notes in Applied \& Computational Mechanics. Springer.

Iverson, R. M. 1997 The physics of debris-flows. Reviews in Geophysics 35, 245-296.

Iverson, R. M. 2003 The debris-flow rheology myth. In Debris-Flow Hazards Mitigation: Mechanics, Prediction and Assessment (ed. D. Rickenmann \& C. L. Chen) pp. 303-314. Millpress. 
Iverson, R. M. 2005 Debris-flow mechanics. In Debris Flow Hazards and Related Phenomena (ed. M. Jakob and O. Hungr), pp. 105-134. Springer-Praxis.

IVERSON R. M. \& Denlinger 2001 Flow of variably fluidized granular masses across threedimensional terrain. Part 1. Coulomb mixture theory. J. Geophys. Res. 106 (B1), 553-566.

Iverson, R. M. \& Vallance, J. W. 2001 New views of granular mass flows. Geology 29 (2), 115-118.

JENKINS, J. T. \& MANCINI, F. 1987 Balance laws and constitutive relations for plane flows of a dense, binary mixture of smooth nearly elastic, circular disks. J. Appl. Mech. 54, 27-34.

Jenkins, J. T. \& Yoon, D. 2001 Segregation in binary mixtures under gravity. Phys. Rev. Lett. 88 (19), 194301-194304.

Jesuthasan, N., Baliga, B. R. \& Savage, S. B. 2006 Use of particle tracking velocimetry for measurements of granular flows: review and application. KONA 24, 15-26.

Johanson, J. R. 1978 Particle segregation ... and what to do about it. Chem. Engng, 183-188.

Jomelli, V. \& Bertran, P. 2001 Wet snow avalanche deposits in the French Alps: structure and sedimentology. Geografis. Annal. A 83 (1-2), 15-28.

Jop, P., Forterre, Y. \& Pouliquen, O. 2005 Crucial role of sidewalls in granular surface flows: consequences for the rheology. J. Fluid Mech. 541, 167-192.

Jop, P., Forterre, Y. \& Pouliquen, O. 2006 A constitutive relation for dense granular flows. Nature 44, 727-730.

Lajeunesse, E., Mangeney-Castelnau, A. \& Vilotte, J. P. 2004 Spreading of a granular mass on a horizontal plane. Phys. Fluids 16, 2371.

Linares-Guerrero, E., Goujon, C. \& Zenit, R. 2007 Increased mobility of bidisperse granular flows. J. Fluid Mech. 593, 475-504.

Lube, G, Huppert, H. E., Sparks, R. S. J. \& Hallworth, M. A. 2004 Axisymmetric collapses of granular columns. J. Fluid Mech. 508, 175-199.

Makse, H. A., Havlin, S., King, P. R. \& Stanley, H. E. 1997 Spontaneous stratification in granular mixtures. Nature 386, 379-382.

McIntyre, M., Rowe, E., Shearer, M., Gray, J. M. N. T. \& Thornton, A. R. 2008 Evolution of a mixing zone in granular avalanches. $A M R X$ 2008, abm008, 1-12.

Middleton, G. V. \& Hampton, M. A. 1976 Subaqueous sediment transport and deposition by sediment gravity flows. In Marine Sediment Transport and Environmental Management (ed. D. J. Stanley \& D. J. P. Swift), pp. 197-218. Wiley.

Möbius, M. E., Lauderdale, B. E., Nagel, S. R. \& JAeger, H. M. 2001 Size segregation of granular particles. Nature 414, 270.

Phillips, J. C., Hogg, A. J., Kerswell, R. R. \& Thomas, N. H. 2006 Enhanced mobility of granular mixtures of fine and coarse particles. Earth Planet. Sci. Lett. 246, 466-480.

Pierson, T. C. 1986 Flow behavior of channelized debris flows, Mount St. Helens, Washington. In Hillslope Processes (ed. A. D. Abrahams), pp. 269-296. Allen \& Unwin.

Pouliquen, O. 1999a Scaling laws in granular flows down rough inclined planes. Phys. Fluids 11 (3), 542-548.

Pouliquen, O. $1999 \mathrm{~b}$ On the shape of granular fronts down rough inclined planes. Phys. Fluids 11(7), 1956-1958.

Pouliquen, O., Delour, J. \& Savage, S. B. 1997 Fingering in granular flows. Nature 386, 816-817.

Pouliquen, O. \& Forterre, Y. 2002 Friction law for dense granular flows: application to the motion of a mass down a rough inclined plane. J. Fluid Mech. 453, 133-151.

Pouliquen, O. \& Vallance, J. W. 1999 Segregation induced instabilities of granular fronts. Chaos $9(3), 621-630$.

Rognon, P. G., Roux, J. N., NaAim, M. \& Chevorr, F. 2007 Dense flows of bidisperse assemblies of disks down an inclined plane. Phys. Fluids 19, 058101.

SAVAGE, S. B. 2008 Free-surface granular flows down heaps. J. Engng Math. 60, 221-240.

SAvage, S. B. \& Hutter, K. 1989 The motion of a finite mass of granular material down a rough incline. J. Fluid Mech. 199, 177-215.

Savage, S. B \& Lun, C. K. K. 1988 Particle size segregation in inclined chute flow of dry cohesionless granular solids. J. Fluid Mech. 189, 311-335.

Shearer, M. Gray, J. M. N. T. \& Thornton, A. R. 2008 Stable solutions of a scalar conservation law for particle-size segregation in dense granular avalanches. Eur. J. Appl. Math. 19, 61-86.

Silbert, L. E., Ertas, D., Grest, G. S., Halsey, T. C., Levine, D. \& Plimpton, S. J. 2001 Granular flow doen an inclined plane: Bagnold scaling and rheology. Phys. Rev. E 64, 051302. 
Silbert, L. E., Landry, J. W. \& Grest, G. S. 2003 Granular flow down a rough inclined plane: transition between thin and thick piles. Phys. Fluids 15 (1), 1-10.

Taberlet, N., Richard, P., Henry, E. \& Delannay, R. 2004 The growth of a super stable heap: an experimental and numerical study. Europhys. Lett. 68 (4), 515-521.

Taberlet, N., Richard, P., Valance, A., Losert, W., Pasini, J. M., Jenkins, J. T. \& Delannay, R. 2003 Superstable granular heap in a thin channel. Phys. Rev. Lett. 91 (26), 264301, 1-4.

Thomas, N. 2000 Reverse and intermediate segregation of large beads in dry granular media. Phys. Rev. E 62 (1), 961-974.

Thornton, A. R. \& Gray, J. M. N. T. 2008 Breaking size-segregation waves and particle recirculation in granular avalanches. J. Fluid Mech. 596, 261-284.

Thornton, A. R., Gray, J. M. N. T. \& HogG, A. J. 2006 A three-phase mixture theory for particle size segregation in shallow granular free-surface flows. J. Fluid Mech. 550, 1-25.

VALLANCE, J. W. 1994 Experimental and field studies related to the behavior of granular mass flows and the characteristics of their deposits. PhD thesis, Michigan Technological University.

Vallance, J. W. 2000 Lahars. In Encyclopedia of Volcanoes (ed. H. Sigurdsson), pp. 601-616. Academic.

Vallance, J. W. \& Savage, S. B. 2000 Particle segregation in granular flows down chutes. In IUTAM Symposium on Segregation in Granular Materials (ed. A. D. Rosato \& D. L. Blackmore), pp. 31-51. Kluwer.

Wieland, M., Gray, J. M. N. T. \& Hutter, K. 1999 Channelised free surface flow of cohesionless granular avalanches in a chute with shallow lateral curvature. J. Fluid Mech. 392, 73-100.

Williams, S. C. 1968 The mixing of dry powders. Powder Technol. 2, 13-20.

Zhang, Y. H. \& Reese, J. M. 2000 The influence of the drag force due to the interstitial gas on granular flows down a chute. Intl J. Multiphase Flow 26, 2049-2072.

Zuriguel, I., Gray, J. M. N. T., Peixinho, J. \& Mullin, T. 2006 Pattern selection by a granular wave in a rotating drum. Phys. Rev. E 73, 061302, 1-4. 